\title{
An experimental and modeling study of the combustion of tetrahydrofuran
}

\author{
Luc-Sy Tran $^{\mathrm{a}, 1}$, Marco Verdicchio ${ }^{\mathrm{a}, 2}$, Fabiola Monge ${ }^{\mathrm{b}}$, Roberto Colino Martin ${ }^{\mathrm{a}}$, Roda Bounaceeur ${ }^{\mathrm{a}}$, \\ Baptiste Sirjean $^{\mathrm{a}}$, Pierre-Alexandre Glaude ${ }^{\mathrm{a}}$, María U. Alzueta ${ }^{\mathrm{b}}$, Frédérique Battin-Leclerc ${ }^{\mathrm{a}, *}$ \\ a Laboratoire Réactions et Génie des Procédés (LRGP), CNRS, Université de Lorraine, ENSIC, 1 rue Grandville, BP 20451, 54001 Nancy Cedex, France \\ ${ }^{\mathrm{b}}$ Aragón Institute of Engineering Research, University of Zaragoza, Mariano Esquillor, 50018 Zaragoza, Spain
}

Tetrahydrofuran (THF) is a well suited starting point fuel to study the combustion chemistry of saturated cyclic esters that are being considered as promising bio-fuels. To better understand the combustion chemistry of THF, laminar low-pressure premixed flame structure, atmospheric adiabatic laminar burning velocities, and high-pressure ignition delay times were investigated. The structure of laminar premixed low-pressure (6.7 kPa) argondiluted (78\%) flames of THF were studied at three equivalence ratios (0.7, 1.0 and 1.3$)$ using on-line gas chromatography analyses. The results consist of temperature and mole fraction profiles (about 40 species) measured as a function of the height above the burner. Ethylene, propene, formaldehyde, acetaldehyde, and dihydrofurans were observed as important interme-diates. Aromatic species were detected in very low amounts. The adiabatic laminar burning velocities of THF-air mixtures were measured using the heat flux burner method at atmospheric pressure (initial tem-peratures from 298 to 398 $\mathrm{K}$, at equivalence ratios from 0.55 to 1.60). The maximum burning velocity of THF was comparable to that of ethanol and diethyl ether. The ignition delay times of THF-oxygen-argon mixtures were measured behind reflected shock waves (temperatures from 1300 to $1700 \mathrm{~K}$, pressures around 8.5 atm, mixtures containing $0.25-1 \%$ of fuel for equivalence ratios of $0.5-2.0$ ). A new detailed kinetic model for THF combustion was developed using a combination of automatic generation (EXGAS), Evans-Polanyi correlations (for H-abstraction kinetic data), and CBS-QB3 theoretical calculations (for unimolecular initiation, $\mathrm{H}$-abstraction and $\beta$-scission kinetic data). An overall good agreement between simulations and the present experimental results has been found. The main THF reaction pathways under flame conditions have been identified from flow rate analyses.

\section{Introduction}

Today there is an increasing interest to shift from fossil fuels to bio-fuels. The use of bio-fuels allows a reduction of the dependence to petroleum-based fuels, and should limit the increase of the total amount of greenhouse gases in the atmosphere. Cyclic ethers of the family of tetrahydrofuran (THF, see molecule structure in Fig. 1), e.g. 2-methyltetrahydrofuran (MTHF) [1] and 2,5-dimethyltetrahydrofuran (DMTHF) [2], have the potential to become promising bio-fuels for internal combustion engines. These fuels have a lower

\footnotetext{
* Corresponding author. Fax: +33 383378120 .

E-mail address: frederique.battin-leclerc@univ-lorraine.fr (F. Battin-Leclerc).

1 Present address: Department of Chemistry, Bielefeld University, Universitätsstraße 25, D-33615 Bielefeld, Germany.

2 Present address: Argonne National Laboratory, Chemical Sciences and Engineering Division, 9700, South Cass Avenue, Building 200, Argonne, IL, USA.
}

heating value ( $\sim 28.5-29.5 \mathrm{MJ} / \mathrm{L})$, which is close to that of gasoline $(\sim 31.6 \mathrm{MJ} / \mathrm{L})$, and higher than that of ethanol $(\sim 21.3 \mathrm{MJ} / \mathrm{L})[3]$. The THF family fuels are second generation bio-fuels and can be produced from non-edible biomass [4-7]. Some tests for MTHF as fuel in engine have been reported relatively early. In 1988, Rudolph and Thomas [8] have comparatively analyzed pollutant emissions from a spark-ignition engine using mixtures of gasoline with $10 \%$ of different potential liquid fuels. Their results show that the MTHF blend has power outputs and emissions of carbon monoxide, nitrogen oxides, and non-methane hydrocarbons close to unleaded gasoline. In 1999, MTHF has been approved by the USDOE as a component of $P$-series fuels for spark-ignition engine. The $P$-series fuels are blends of ethanol (25-40\% by vol.), MTHF (20-35\%), and $\mathrm{C}_{+5}$ alkanes (25-40\%), with butane (0-10\%) added to blends, that would be used in severe cold-weather conditions to meet engine cold start requirements $[9,10]$. 
In addition, THF and other saturated cyclic ethers have also been identified among the emissions produced during the lowtemperature oxidation and auto-ignition of alkanes, this formation being due to isomerizations of alkylhydroperoxy radicals $[11,12]$. The subsequent reactions of these cyclic ethers can then influence the overall chemical kinetic mechanisms of the oxidation of alkanes. Therefore, a better understanding of saturated cyclic ether combustion chemistry, starting by that of THF, is necessary, and should be gained before actually using these fuels in engines.

The pyrolysis of THF was first experimentally studied in the 1950s [13,14] and then in 1986 by Lifshitz et al. [15] behind reflected shock waves (temperatures between 1070 and $1530 \mathrm{~K}$ at pressures around 3 bar). More recently Verdicchio et al. [16] have studied the unimolecular decompositions of THF using quantum chemistry and reaction rate theory. These computed reactions have been used in a pyrolysis model which satisfactorily reproduces the results of Lifshitz et al. [15] showing a determinant importance of the reactions involving carbenes and diradicals under these conditions.

A few studies on the combustion of THF have also been carried out. The low-temperature (493 K) oxidation of THF was investigated by Molera et al. [17], in a static reactor. A motored engine study of auto-ignition chemistry of acyclic and cyclic ethers, including THF, was performed by Leppard [18]. The chemical mechanisms responsible for auto-ignition of both ether classes are detailed and can be used to explain the differences in antiknock characteristics. Ignition delay times and oxidation of THF have been studied by Dagaut et al. [19] behind reflected shock waves and in a jet-stirred reactor (JSR), respectively (temperatures from 800 to $1800 \mathrm{~K}$, pressures from 202 to $1013 \mathrm{kPa}$, equivalence ratio $(\varphi)$ from 0.5 to 2$)$. On the basis of these results, a detailed reaction mechanism with 71 species and 484 reactions was proposed. The JSR study showed that a large amount of aldehydes (formaldehyde, acetaldehyde, and propanal) were produced during the THF oxidation. Recently, Kasper et al. [20] have investigated the structure of laminar premixed low-pressure THF flames using photoionization (PI) and electron-ionization (EI) molecular-beam mass spectrometry (MBMS). About 60 intermediates including radicals have been
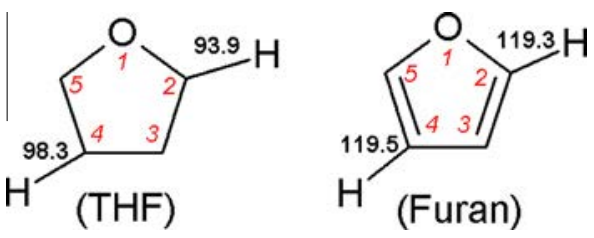

Fig. 1. Structure of THF compared with that of furan. Italic number near the atom: atom label; bold numbers: calculated bond energy (in $\mathrm{kcal} \mathrm{mol}^{-1}$ ) from the thermochemical data of molecules and radicals calculated theoretically at the CBSQB3 level of theory in present and work [52]. measured and analyzed. Finally Uygun et al. [21] have studied ignition delay times of THF in a high pressure shock tube at temperatures from 691 to $1100 \mathrm{~K}$ and pressures of 20 and 40 bar for stoichiometric mixtures. No model has been proposed to support these two recent experimental work $[20,21]$. Theoretical calculations have also been performed [2,22] to study the kinetics and thermochemistry of THF and its derivatives.

The main experimental studies published in the literature on the pyrolysis and combustion of THF are summarized in Table 1. In the present work, as part of a continuing effort to improve the knowledge on the combustion chemistry of this cyclic ether, we report experimental data obtained in premixed flames and in shock tube. These data consist of: (i) temperature and mole fraction profiles of chemical species measured in low-pressure premixed THF flames including isomer identification, (ii) laminar burning velocities obtained under atmospheric pressure using the heat flux method, and (iii) ignition delay times measured in a shock tube behind reflected shock waves at temperatures higher than those investigated by Uygun et al. [21]. A new detailed kinetic model for THF combustion has been developed using a combination of automatic generation (EXGAS), Evans-Polanyi correlation, and CBS-QB3 theoretical calculations in order to represent the present high-temperature experimental results. This model includes the unimolecular initiations recently studied by Verdicchio et al. [16].

\section{Experimental and simulation methods}

The combustion chemistry of THF was studied in low-pressure and atmospheric flat flames, and in a shock tube. THF liquid fuel (>99.7\% pure) was supplied by VWR. This section presents the experimental and simulation methods used in the present study. In the flame experiments, gas flow rates (of oxygen, argon and nitrogen) were measured using Bronkhorst High-Tech Mass Flow Controllers (MFC) and flow rates of liquid THF were measured using Bronkhorst mini-CORI-FLOW Mass Flow Controller connected to a Controlled Evaporator Mixer (CEM). The liquid fuel was mixed with inert gas and then evaporated by passing through the CEM set at $373 \mathrm{~K}$. The gas and liquid mass flow accuracy was $\pm 0.5 \%$.

\subsection{Low-pressure premixed flat flame structure}

Three flames, fuel-lean ( $\varphi=0.7)$, stoichiometric $(\varphi=1.0)$, and fuel-rich $(\varphi=1.3)$, were investigated at 50 Torr $(6.7 \mathrm{kPa})$ with an argon dilution of $78 \%$. The experimental setup developed in LRGP to study stable species profiles in a laminar premixed flat flame at low-pressure has been described previously [23-25]. Briefly, all flames are stabilized on a McKenna burner (diameter of $60 \mathrm{~mm}$ ) housed in a vacuum chamber. The burner is cooled with

Table 1

Recent main studies on the pyrolysis and combustion of THF.

\begin{tabular}{|c|c|c|c|c|}
\hline \multirow[t]{2}{*}{ Reactor } & \multicolumn{3}{|c|}{ Operating conditions } & \multirow[t]{2}{*}{ Ref. } \\
\hline & $T(\mathrm{~K})$ & $P(\mathrm{kPa})$ & $\varphi$ & \\
\hline ST & $1070-1530$ & $178-1018$ & Pyrolysis & Lifshitz et al. [15] \\
\hline SR & 493 & 21 & 2.75 & Molera et al. [17] \\
\hline ICE & $400^{\mathrm{a}} ; 827^{\mathrm{b}}$ & $80^{\mathrm{a}} ; 1200^{\mathrm{b}}$ & $0.95-1.0$ & Leppard [18] \\
\hline ST & $1000-180$ & $200-500$ & $0.5-2.0$ & Dagaut et al. [19] \\
\hline JSR & $800-1100$ & 1000 & $0.5-1.0$ & (a 71 species and 484 reactions model has been proposed) \\
\hline FF & $500-2300$ & $2.0-3.3$ & $1.0-1.75$ & Kasper et al. [20] \\
\hline ST & $691-1100$ & $2000-5000$ & 1.0 & Uygun et al. [21] \\
\hline
\end{tabular}

SR - static reactor; ST - shock tube; ICE - internal combustion engine; JSR - jet-stirred reactor; FF - flat flame.

a In admission collector.

b Maximal value in cylinder at compression ratio of 8.7. 
Table 2

Low-pressure flame initial operating conditions.

\begin{tabular}{|c|c|c|c|c|c|c|c|}
\hline \multirow[t]{2}{*}{$\varphi^{\mathrm{a}}$} & \multicolumn{3}{|c|}{ Gas flow (NL/min) } & \multirow[t]{2}{*}{ Dilution $^{\mathrm{b}}(\%)$} & \multirow[t]{2}{*}{$\mathrm{C} / \mathrm{O}$ ratio } & \multirow[t]{2}{*}{ Pressure (kPa) } & \multirow[t]{2}{*}{ Gas velocity at the burner surface ${ }^{\mathrm{c}}\left(\mathrm{cm} \mathrm{s}^{-1}\right)$} \\
\hline & THF (gas) & $\mathrm{O}_{2}$ & $\mathrm{Ar}$ & & & & \\
\hline 0.7 & 0.166 & 1.30 & 4.62 & 78 & 0.24 & 6.7 & 67 \\
\hline 1.0 & 0.234 & 1.29 & 4.56 & 78 & 0.33 & 6.7 & 67 \\
\hline 1.3 & 0.301 & 1.27 & 4.51 & 78 & 0.42 & 6.7 & 67 \\
\hline
\end{tabular}

a Equivalence ratio.

b Dilution $=\mathrm{Ar} /\left(\mathrm{Ar}+\mathrm{O}_{2}\right)$.

c Temperature of $333 \mathrm{~K}$.

water at a constant temperature of $333 \mathrm{~K}$. The initial operating conditions related to THF low-pressure flames are summarized in Table 2.

Analyses were made using gas chromatography (GC) via a heated (at $423 \mathrm{~K}$ ) on-line connection to a quartz probe. Three gas chromatographs were used. These were equipped with three types of columns: Carbosphere, HP-Plot Q and HP-Molsieve, and three types of detectors: a flame ionization detector (FID) coupled with methanizer, a thermal conductivity detector (TCD), and a mass spectrometry (MS). The HP-Plot Q column with helium as carrier gas was used to analyze $\mathrm{CO}_{2}$, all the $\mathrm{C}_{2+}$ hydrocarbon and oxygenated species by FID. This column was also used to analyze $\mathrm{H}_{2} \mathrm{O}$ by TCD. The HP-Molsieve column with helium as the carrier gas was used to analyze $\mathrm{CH}_{4}$ and $\mathrm{CO}$ by FID and Ar by TCD. In our previous work [23], $\mathrm{CO}$ and $\mathrm{CO}_{2}$ can only be detected by TCD, and formaldehyde cannot be detected. Here, $\mathrm{CO}$ and $\mathrm{CO}_{2}$, as well as formaldehyde were converted to methane by the methanizer, and could then be detected by FID, which is more sensitive (by a factor of $100)$ than TCD. Stable species were identified by the determination of their individual retention times and by mass spectrometry (GC-MS). Calibrations were made directly using cold-gas mixtures. For species for which a direct calibration procedure is not applicable, the calibration factors were estimated thanks to the hydrogenation in the methanizer, considering that this species has been transformed into the alkane involving the same number of carbon atoms. The calculated uncertainties on the mole fraction measurements of the quantified species were $\sim 5 \%$ for the major compounds and $\sim 10 \%$ for minor products $(<100 \mathrm{ppm})$. The FID detection threshold was about $1 \mathrm{ppm}$, while the TCD detection limit was about $50 \mathrm{ppm}$ for $\mathrm{H}_{2} \mathrm{O}, \mathrm{H}_{2}$ and $\mathrm{O}_{2}$. Note that studies made under the same conditions for furan has shown that a general good agreement between EI-MBMS and GC measurements could be spotted for the maximum mole fraction of the analyzed species, within the experimental error limits [25].

Flame temperature profiles were obtained using a PtRh (6\%)PtRh (30\%) type B thermocouple (diameter $100 \mu \mathrm{m})$. The thermocouple wire was supported by an arm and crossed the flame horizontally to avoid conduction heat losses. The thermocouple junction was located at the center of the burner. In order to prevent catalytic effects when placed in a flame, the thermocouple was coated with a ceramic layer of $\mathrm{BeO}-\mathrm{Y}_{2} \mathrm{O}_{3}$ [26] which was obtained by dipping the thermocouple in a hot solution of $\mathrm{Y}_{2}\left(\mathrm{CO}_{3}\right)_{3}(93 \%$ mass) and $\mathrm{BeO}$ (7\% mass) followed by drying in a Meker burner flame. Radiative heat losses were corrected using the electrical compensation method [26]. Uncertainty on the measurement of temperature was about $\pm 100 \mathrm{~K}$ in the burned gases. Note that as discussed by Tran et al. [27], the uncertainty on temperature has only a limited impact on measured maximum species mole fractions. In the fresh gases, there was an uncertainty of $\pm 0.05 \mathrm{~mm}$ on the position of the temperature profile in the direction perpendicular to the burner surface. A sighting telescope (cathetometer) measured the position of the burner relative to the probe or the thermocouple with an accuracy of $0.01 \mathrm{~mm}$.

\subsection{Laminar burning velocities measurements in atmospheric flat flame}

Laminar burning velocities were measured using the heat flux burner method [28] applied to a flat flame adiabatic burner recently built in LRGP, which has already been described previously $[29,30]$. The apparatus consisted of a brass burner plate ( $2 \mathrm{~mm}$ thick and $30 \mathrm{~mm}$ in diameter) mounted on a plenum chamber. The plate was perforated with small holes of diameter $0.5 \mathrm{~mm}$, with the pitch between them being $0.7 \mathrm{~mm}$. Eight type $\mathrm{K}$ thermocouples $(0.5 \mathrm{~mm}$ diameter) were soldered into the plate surface and were located at different distances and angles from the center to the periphery of the burner. The plenum chamber was encompassed by a thermostatic oil jacket, the temperature of which was set to the desired initial temperature of the unburned gas mixture. The circumference of the burner plate was heated with thermostatic oil set to $50 \mathrm{~K}$ above the temperature of the unburned gas mixture. In practice, if an initial temperature of $398 \mathrm{~K}$ was desired, the temperature of the plenum chamber was set to $398 \mathrm{~K}$ and that of the burner plate to $448 \mathrm{~K}$. Thus, the heat gain of the unburned gas mixture can compensate for the heat loss necessary for stabilizing the flame, knowing that monitoring of the heat loss or gain was performed with the thermocouples.

The error estimation in the laminar burning velocity was described in detail in [29]. An estimated global error in the laminar burning velocity is about $4 \%$. In addition, the error in the calculation of equivalence ratios is about $1 \%$. This results mainly from the error in the mass flow measurements for oxygen and liquid fuel.

In the present study, the measurements of laminar burning velocity were performed under atmospheric pressure $(101.3 \mathrm{kPa})$, in a range of temperatures from 298 to $398 \mathrm{~K}$, and at equivalence ratios from 0.55 to 1.60 .

\subsection{Ignition delay times in shock tube}

The measurements of ignition delay times were performed using a shock tube in LRGP, which has already been described previously $[31,32]$. In brief, the stainless steel shock tube included a reaction and a driver parts separated by two terphane diaphragms, which were ruptured by decreasing suddenly the pressure in the space separating them. The reaction and the driver parts were respectively 400.6 and $89 \mathrm{~cm}$ in length. The driver gas was helium. The incident and reflected shock velocities were measured by four piezo-electric pressure transducers located along the reaction section. The pressure and temperature of the test gas behind the reflected shock wave were derived from the values of the initial pressure in the low pressure section and of the incident shock velocity by using ideal one-dimensional shock equations. The error on the temperature was about $20 \mathrm{~K}$. The onset of ignition was detected by excited $\mathrm{OH}^{*}$ radical emission at $306 \mathrm{~nm}$ through a quartz window with a photomultiplier fitted with a monochromator at the end of the reaction part. The quartz window was located 
Table 3

Primary mechanism of the high-temperature pyrolysis and oxidation of THF. The rate constants are given $\left(k=A T^{n} \exp \left(-E_{a} / \mathrm{RT}\right)\right)$ in $\mathrm{cm}^{3}$, mol, $\mathrm{s}, \mathrm{cal}$ units.

\begin{tabular}{|c|c|c|c|c|c|}
\hline Reactions & $A$ & $n$ & $E_{a}$ & Footnote & $\mathrm{n}^{\circ}$ \\
\hline \multicolumn{6}{|l|}{ Reactions of $\mathrm{THF}\left(\mathrm{C}_{4} \mathrm{H}_{8} \mathrm{O}\right)$} \\
\hline \multicolumn{6}{|l|}{ Unimolecular initiations } \\
\hline $\mathrm{H}+\mathrm{THF}-\mathrm{yl}-3=\mathrm{THF}$ & $1.00 \times 10^{+14}$ & 0.0 & 0.0 & a & 1 \\
\hline $\mathrm{H}+\mathrm{THF}-\mathrm{yl}-2=\mathrm{THF}$ & $1.00 \times 10^{+14}$ & 0.0 & 0.0 & a & 2 \\
\hline \multirow{3}{*}{$\mathrm{THF}=\mathrm{CH}_{2} \mathrm{CHCH}_{2} \mathrm{OCH}_{3}$} & $5.22 \times 10^{+65}$ & -14.81 & 119,750 & $0.1 \mathrm{~atm}^{\mathrm{b}}$ & 3 \\
\hline & $4.82 \times 10^{+49}$ & -10.05 & 111,690 & $1 \mathrm{~atm}^{\mathrm{b}}$ & \\
\hline & $9.88 \times 10^{+33}$ & -5.47 & 102,820 & $10 \mathrm{~atm}^{\mathrm{b}}$ & \\
\hline \multirow[t]{3}{*}{$\mathrm{THF}=\mathrm{CH}_{2} \mathrm{CHOCH}_{2} \mathrm{CH}_{3}$} & $2.72 \times 10^{+65}$ & -14.69 & 120,150 & $0.1 \mathrm{~atm}^{\mathrm{b}}$ & 4 \\
\hline & $1.77 \times 10^{+51}$ & -10.44 & 113,500 & $1 \mathrm{~atm}^{\mathrm{b}}$ & \\
\hline & $7.75 \times 10^{+34}$ & -5.66 & 104,280 & $10 \mathrm{~atm}^{\mathrm{b}}$ & \\
\hline $\mathrm{THF}=\mathrm{C}_{3} \mathrm{H}_{7} \mathrm{CHO}$ & $2.62 \times 10^{+61}$ & -13.71 & 110,910 & $0.1 \mathrm{~atm}^{\mathrm{b}}$ & \\
\hline \multirow[t]{2}{*}{ (reactions of butanal $\left(\mathrm{C}_{3} \mathrm{H}_{7} \mathrm{CHO}\right)$ are in the reaction base) } & $5.56 \times 10^{+46}$ & -9.42 & 102,910 & $1 \mathrm{~atm}^{\mathrm{b}}$ & 5 \\
\hline & $1.06 \times 10^{+35}$ & -6.00 & 96,029 & 10 atm $^{\mathrm{b}}$ & \\
\hline \multirow[t]{3}{*}{$\mathrm{THF}=\mathrm{CH}_{2} \mathrm{CHCH}_{2} \mathrm{CH}_{2} \mathrm{OH}$} & $7.79 \times 10^{+57}$ & -12.60 & 108,610 & $0.1 \mathrm{~atm}^{\mathrm{b}}$ & 6 \\
\hline & $1.65 \times 10^{+41}$ & -7.71 & 99,453 & $1 \mathrm{~atm}^{\mathrm{b}}$ & \\
\hline & $3.68 \times 10^{+27}$ & -3.74 & 91,549 & 10 atm $^{\mathrm{b}}$ & \\
\hline \multirow[t]{3}{*}{$\mathrm{THF}=\mathrm{C}_{3} \mathrm{H}_{6}+\mathrm{CH}_{2} \mathrm{O}$} & $5.76 \times 10^{+50}$ & -10.43 & 104,950 & $0.1 \mathrm{~atm}^{\mathrm{b}}$ & 7 \\
\hline & $2.17 \times 10^{+35}$ & -5.81 & 97,786 & $1 \mathrm{~atm}^{\mathrm{b}}$ & \\
\hline & $1.08 \times 10^{+26}$ & -2.96 & 95,111 & 10 atm $^{\mathrm{b}}$ & \\
\hline \multirow[t]{3}{*}{$\mathrm{THF}=\mathrm{c}-\mathrm{CH}_{2} \mathrm{OCH}_{2}+\mathrm{C}_{2} \mathrm{H}_{4}$} & $2.64 \times 10^{+81}$ & -19.06 & 138,460 & $0.1 \mathrm{~atm}^{\mathrm{b}}$ & 8 \\
\hline & $9.25 \times 10^{+64}$ & -14.06 & 131,310 & $1 \mathrm{~atm}^{\mathrm{b}}$ & \\
\hline & $1.27 \times 10^{+46}$ & -8.50 & 121,300 & 10 atm $^{\mathrm{b}}$ & \\
\hline $\mathrm{THF}=\mathrm{C}_{4} \mathrm{H}_{6} \mathrm{O}-1+\mathrm{H}_{2}$ & $8.13 \times 10^{+52}$ & -11.43 & 103,770 & $0.1 \mathrm{~atm}^{\mathrm{b}}$ & 9 \\
\hline \multirow{2}{*}{$\left(\mathrm{C}_{4} \mathrm{H}_{6} \mathrm{O}-1\right.$ is a cyclic diradical $)$} & $3.86 \times 10^{+36}$ & -6.65 & 94,752 & $1 \mathrm{~atm}^{\mathrm{b}}$ & \\
\hline & $8.05 \times 10^{+22}$ & -2.68 & 86,765 & $10 \mathrm{~atm}^{\mathrm{b}}$ & \\
\hline \multicolumn{6}{|l|}{ Reactions of cyclic diradicals produced from unimolecular initiations } \\
\hline $\mathrm{C}_{4} \mathrm{H}_{6} \mathrm{O}-1=\mathrm{CH}_{2} \mathrm{CHOCHCH}_{2}$ & $2.16 \times 10^{+13}$ & -0.024 & 25,895 & b & 10 \\
\hline $\mathrm{C}_{4} \mathrm{H}_{6} \mathrm{O}-1=\mathrm{C}_{4} \mathrm{H}_{6} \mathrm{O}-3$ & $1.58 \times 10^{+12}$ & 0.412 & 54,729 & b & 11 \\
\hline $\mathrm{C}_{4} \mathrm{H}_{6} \mathrm{O}-1=2,3-\mathrm{DHF}$ & $6.27 \times 10^{+11}$ & 0.3 & 18,953 & b & 12 \\
\hline $\mathrm{C}_{4} \mathrm{H}_{6} \mathrm{O}-1=\mathrm{CA} 5$ & $1.32 \times 10^{+12}$ & 0.277 & 40,449 & b & 13 \\
\hline $\mathrm{C}_{4} \mathrm{H}_{6} \mathrm{O}-1=\mathrm{CO}+\mathrm{C}_{3} \mathrm{H}_{6}$ & $8.88 \times 10^{+12}$ & 0.415 & 78,893 & b & 14 \\
\hline $\mathrm{C}_{4} \mathrm{H}_{6} \mathrm{O}-3=2,5-\mathrm{DHF}$ & $4.23 \times 10^{+12}$ & -0.078 & 14,164 & b & 15 \\
\hline $\mathrm{C}_{4} \mathrm{H}_{6} \mathrm{O}-3=\mathrm{CH}_{2} \mathrm{CHCH}_{2} \mathrm{CHO}$ & $4.30 \times 10^{+12}$ & -0.078 & 4464 & b & 16 \\
\hline $\mathrm{CA} 5=\mathrm{C}_{2} \mathrm{H}_{4}+\mathrm{CH}_{2} \mathrm{CO}$ & $7.04 \times 10^{+12}$ & 0.223 & 16,106 & b & 17 \\
\hline $\mathrm{CA} 5=\mathrm{CO}+\mathrm{C}_{3} \mathrm{H}_{6}$ & $7.10 \times 10^{+13}$ & 0.506 & 35,628 & b & 18 \\
\hline$\left(\mathrm{C}_{4} \mathrm{H}_{6} \mathrm{O}-3\right.$ is a cyclic diradical, $\mathrm{CA} 5$ is a carbene) & & & & & \\
\hline Bimolecular initiations & & & & & \\
\hline $\mathrm{THF}+\mathrm{O}_{2} \Rightarrow \mathrm{THF}-\mathrm{yl}-2+\mathrm{OOH}$ & $4.00 \times 10^{+13}$ & 0.0 & 46,247 & c & 19 \\
\hline $\mathrm{THF}+\mathrm{O}_{2} \Rightarrow \mathrm{THF}-\mathrm{yl}-3+\mathrm{OOH}$ & $4.00 \times 10^{+13}$ & 0.0 & 52,947 & c & 20 \\
\hline H-abstractions & & & & & \\
\hline $\mathrm{THF}+\mathrm{O} \Rightarrow \mathrm{THF}-\mathrm{yl}-2+\mathrm{OH}$ & $6.80 \times 10^{+08}$ & 1.5 & 385 & d & 21 \\
\hline $\mathrm{THF}+\mathrm{O} \Rightarrow \mathrm{THF}-\mathrm{yl}-3+\mathrm{OH}$ & $6.80 \times 10^{+08}$ & 1.5 & 3678 & d & 22 \\
\hline $\mathrm{THF}+\mathrm{H} \Rightarrow \mathrm{THF}-\mathrm{yl}-2+\mathrm{H}_{2}$ & $1.16 \times 10^{+07}$ & 2.02 & 3204 & e & 23 \\
\hline $\mathrm{THF}+\mathrm{H} \Rightarrow \mathrm{THF}-\mathrm{yl}-3+\mathrm{H}_{2}$ & $1.27 \times 10^{+04}$ & 2.85 & 4185 & e & 24 \\
\hline $\mathrm{THF}+\mathrm{OH} \Rightarrow \mathrm{THF}-\mathrm{yl}-2+\mathrm{H}_{2} \mathrm{O}$ & $4.80 \times 10^{+06}$ & 2.0 & -2630 & d & 25 \\
\hline $\mathrm{THF}+\mathrm{OH} \Rightarrow \mathrm{THF}-\mathrm{yl}-3+\mathrm{H}_{2} \mathrm{O}$ & $4.80 \times 10^{+06}$ & 2.0 & -435 & d & 26 \\
\hline $\mathrm{THF}+\mathrm{OOH} \Rightarrow \mathrm{THF}-\mathrm{yl}-2+\mathrm{H}_{2} \mathrm{O}_{2}$ & $5.60 \times 10^{+04}$ & 2.69 & 15,348 & d & 27 \\
\hline $\mathrm{THF}+\mathrm{OOH} \Rightarrow \mathrm{THF}-\mathrm{yl}-3+\mathrm{H}_{2} \mathrm{O}_{2}$ & $5.60 \times 10^{+04}$ & 2.69 & 17,982 & d & 28 \\
\hline $\mathrm{THF}+\mathrm{CH}_{3} \Rightarrow \mathrm{THF}-\mathrm{yl}-2+\mathrm{CH}_{4}$ & $8.37 \times 10^{-04}$ & 4.6 & 4114 & e & 29 \\
\hline $\mathrm{THF}+\mathrm{CH}_{3} \Rightarrow \mathrm{THF}-\mathrm{yl}-3+\mathrm{CH}_{4}$ & $4.27 \times 10^{-06}$ & 5.26 & 4379 & e & 30 \\
\hline $\mathrm{THF}+\mathrm{CHO} \Rightarrow \mathrm{THF}-\mathrm{yl}-2+\mathrm{HCHO}$ & $2.20 \times 10^{+07}$ & 1.9 & 17,000 & c & 31 \\
\hline $\mathrm{THF}+\mathrm{CHO} \Rightarrow \mathrm{THF}-\mathrm{yl}-3+\mathrm{HCHO}$ & $2.20 \times 10^{+07}$ & 1.9 & 17,000 & c & 32 \\
\hline $\mathrm{THF}+\mathrm{CH}_{2} \mathrm{OH} \Rightarrow$ THF-yl- $2+\mathrm{CH}_{3} \mathrm{OH}$ & $1.20 \times 10^{+02}$ & 3.0 & 12,000 & c & 33 \\
\hline $\mathrm{THF}+\mathrm{CH}_{2} \mathrm{OH} \Rightarrow \mathrm{THF}-\mathrm{yl}-3+\mathrm{CH}_{3} \mathrm{OH}$ & $1.20 \times 10^{+02}$ & 3.0 & 12,000 & c & 34 \\
\hline $\mathrm{THF}+\mathrm{CH}_{3} \mathrm{O} \Rightarrow$ THF-yl-2 $+\mathrm{CH}_{3} \mathrm{OH}$ & $2.90 \times 10^{+11}$ & 0.0 & 4500 & c & 35 \\
\hline $\mathrm{THF}+\mathrm{CH}_{3} \mathrm{O} \Rightarrow \mathrm{THF}-\mathrm{yl}-3+\mathrm{CH}_{3} \mathrm{OH}$ & $2.90 \times 10^{+11}$ & 0.0 & 4500 & c & 36 \\
\hline $\mathrm{THF}+\mathrm{CH}_{3} \mathrm{OO} \Rightarrow \mathrm{THF}-\mathrm{yl}-2+\mathrm{CH}_{3} \mathrm{OOH}$ & $6.00 \times 10^{+12}$ & 0.0 & 17,500 & c & 37 \\
\hline $\mathrm{THF}+\mathrm{CH}_{3} \mathrm{OO} \Rightarrow \mathrm{THF}-\mathrm{yl}-3+\mathrm{CH}_{3} \mathrm{OOH}$ & $6.00 \times 10^{+12}$ & 0.0 & 17,500 & c & 38 \\
\hline $\mathrm{THF}+\mathrm{C}_{2} \mathrm{H}_{5} \Rightarrow \mathrm{THF}-\mathrm{yl}-2+\mathrm{C}_{2} \mathrm{H}_{6}$ & $4.00 \times 10^{+11}$ & 0.0 & 11,000 & c & 39 \\
\hline $\mathrm{THF}+\mathrm{C}_{2} \mathrm{H}_{5} \Rightarrow$ THF-yl- $3+\mathrm{C}_{2} \mathrm{H}_{6}$ & $4.00 \times 10^{+11}$ & 0.0 & 11,000 & c & 40 \\
\hline Reactions of THF-yl-2 $\left(\mathrm{C}_{4} \mathrm{H}_{7} \mathrm{O}\right)$ and subsequently derived radi & & & & & \\
\hline THF-yl-2 = THF-yl-3 & $3.19 \times 10^{-01}$ & 3.85 & 35,800 & e & 41 \\
\hline THF-yl-2 $\Rightarrow \mathrm{R} 5 \mathrm{C}_{4} \mathrm{H}_{7} \mathrm{OA}$ & $2.54 \times 10^{+10}$ & 0.8 & 21,585 & f & 42 \\
\hline THF-yl-2 $\Rightarrow \mathrm{R} 1 \mathrm{C}_{4} \mathrm{H}_{7} \mathrm{OEZ}$ & $3.14 \times 10^{+10}$ & 0.95 & 32,395 & e & 43 \\
\hline THF-yl-2 $=2,3-\mathrm{DHF}+\mathrm{H}$ & $2.80 \times 10^{+04}$ & 2.7 & 45,675 & e & 44 \\
\hline 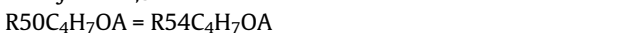 & $1.30 \times 10^{+01}$ & 3.08 & 25,615 & e & 45 \\
\hline 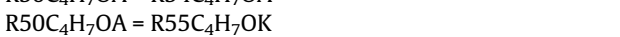 & $1.15 \times 10^{+04}$ & 2.22 & 12,675 & e & 46 \\
\hline $\mathrm{R} 0 \mathrm{C}_{4} \mathrm{H}_{7} \mathrm{OA} \Rightarrow \mathrm{CH}_{2} \mathrm{CHO}+\mathrm{C}_{2} \mathrm{H}_{4}$ & $3.34 \times 10^{+10}$ & 0.85 & 21,269 & e & 47 \\
\hline $\mathrm{R} 1 \mathrm{C}_{4} \mathrm{H}_{7} \mathrm{OEZ}=\mathrm{R}_{5} 6 \mathrm{C}_{4} \mathrm{H}_{7} \mathrm{OEV}$ & $2.90 \times 10^{+08}$ & 1.0 & 16,940 & e & 48 \\
\hline 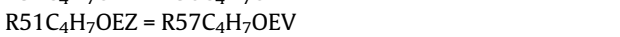 & $5.70 \times 10^{+08}$ & 1.0 & 14,040 & e & 49 \\
\hline 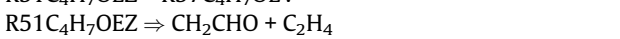 & $9.82 \times 10^{+09}$ & 0.93 & 17,133 & e & 50 \\
\hline $\mathrm{R}_{5} 4 \mathrm{C}_{4} \mathrm{H}_{7} \mathrm{OA} \Rightarrow \mathrm{CH}_{3}+\mathrm{C}_{2} \mathrm{H}_{3} \mathrm{CHO}$ & $2.00 \times 10^{+13}$ & 0.0 & 31,000 & c & 51 \\
\hline
\end{tabular}


Table 3 (continued)

\begin{tabular}{|c|c|c|c|c|c|}
\hline Reactions & $A$ & $n$ & $E_{a}$ & Footnote & $\mathrm{n}^{\circ}$ \\
\hline 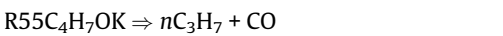 & $3.65 \times 10^{+12}$ & 0.6 & 15,123 & e & 52 \\
\hline $\mathrm{R} 56 \mathrm{C}_{4} \mathrm{H}_{7} \mathrm{OEV}=\mathrm{R} 1 \mathrm{C}_{4} \mathrm{H}_{7} \mathrm{OEZ}$ & $3.30 \times 10^{+09}$ & 1.0 & 42,640 & c & 53 \\
\hline 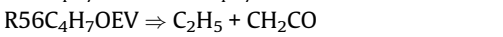 & $8.50 \times 10^{+12}$ & 0.0 & 28,000 & c & 54 \\
\hline 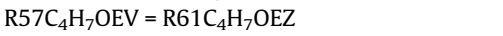 & $3.30 \times 10^{+09}$ & 1.0 & 16,540 & c & 55 \\
\hline $\mathrm{R}^{2} 7 \mathrm{C}_{4} \mathrm{H}_{7} \mathrm{OEV} \Rightarrow \mathrm{R} 15 \mathrm{C}_{2} \mathrm{H}_{5} \mathrm{O}+\mathrm{C}_{2} \mathrm{H}_{2}$ & $8.50 \times 10^{+12}$ & 0.0 & 28,000 & c & 56 \\
\hline 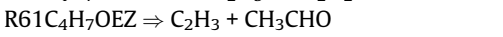 & $2.00 \times 10^{+13}$ & 0.0 & 24,000 & c & 57 \\
\hline THF-yl-2 $+\mathrm{CH}_{3}=\mathrm{MTHF}$ & $5.00 \times 10^{+12}$ & 0.0 & 0.0 & c & 58 \\
\hline \multicolumn{6}{|c|}{ Reactions of THF-yl-3 $\left(\mathrm{C}_{4} \mathrm{H}_{7} \mathrm{O}\right)$ and subsequently derived radicals } \\
\hline THF-yl-3 $\Rightarrow \mathrm{R}_{5} 2 \mathrm{C}_{4} \mathrm{H}_{7} \mathrm{OEZ}$ & $9.01 \times 10^{+10}$ & 0.71 & 32,366 & e & 59 \\
\hline THF-yl-3 $\Rightarrow \mathrm{R}_{5} 3 \mathrm{C}_{4} \mathrm{H}_{7} \mathrm{OZ}$ & $2.73 \times 10^{+11}$ & 0.46 & 30,540 & f & 60 \\
\hline THF-yl-3 $=2,3-\mathrm{DHF}+\mathrm{H}$ & $9.18 \times 10^{+09}$ & 1.1 & 32,897 & e & 61 \\
\hline THF-yl-3 = 2,5-DHF + H & $1.65 \times 10^{+10}$ & 1.1 & 35,717 & e & 62 \\
\hline $\mathrm{R}_{52} \mathrm{C}_{4} \mathrm{H}_{7} \mathrm{OEZ}=\mathrm{R}_{58} \mathrm{C}_{4} \mathrm{H}_{7} \mathrm{OEX}$ & $1.09 \times 10^{-32}$ & 12.4 & 8730 & e & 63 \\
\hline $\mathrm{R}_{52} \mathrm{C}_{4} \mathrm{H}_{7} \mathrm{OEZ}=\mathrm{R}_{5} 9 \mathrm{C}_{4} \mathrm{H}_{7} \mathrm{OEV}$ & $2.90 \times 10^{+08}$ & 1.0 & 24,940 & c & 64 \\
\hline $\mathrm{R} 52 \mathrm{C}_{4} \mathrm{H}_{7} \mathrm{OEZ}=\mathrm{R}_{60 C_{4}} \mathrm{H}_{7} \mathrm{OEV}$ & $5.70 \times 10^{+08}$ & 1.0 & 20,040 & c & 65 \\
\hline $\mathrm{R}_{52} \mathrm{C}_{4} \mathrm{H}_{7} \mathrm{OEZ} \Rightarrow \mathrm{C}_{3} \mathrm{H}_{5}-\mathrm{Y}+\mathrm{HCHO}$ & $9.78 \times 10^{+07}$ & 1.5 & 15,115 & e & 66 \\
\hline $\mathrm{R}_{53} \mathrm{C}_{4} \mathrm{H}_{7} \mathrm{OZ} \Rightarrow \mathrm{C}_{3} \mathrm{H}_{5}-\mathrm{Y}+\mathrm{HCHO}$ & $1.36 \times 10^{+11}$ & 0.65 & 6337 & e & 67 \\
\hline 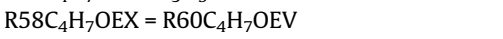 & $1.90 \times 10^{+10}$ & 1.0 & 52,300 & c & 68 \\
\hline 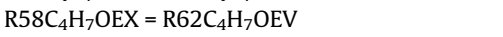 & $9.70 \times 10^{+09}$ & 1.0 & 44,300 & e & 69 \\
\hline 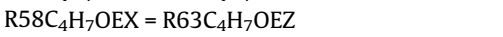 & $6.18 \times 10^{-09}$ & 5.79 & 19,600 & e & 70 \\
\hline $\mathrm{R} 58 \mathrm{C}_{4} \mathrm{H}_{7} \mathrm{OEX} \Rightarrow \mathrm{CH}_{3}+\mathrm{C}_{2} \mathrm{H}_{3} \mathrm{CHO}$ & $4.30 \times 10^{+12}$ & 0.0 & 33,200 & c & 71 \\
\hline $\mathrm{R} 58 \mathrm{C}_{4} \mathrm{H}_{7} \mathrm{OEX}+\mathrm{O}_{2} \Rightarrow \mathrm{C}_{4} \mathrm{H}_{6} \mathrm{OZ2}+\mathrm{OOH}$ & $1.00 \times 10^{+12}$ & 0.0 & 22,730 & c & 72 \\
\hline 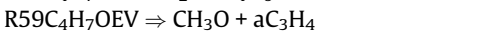 & $8.50 \times 10^{+12}$ & 0.0 & 28,000 & c & 73 \\
\hline 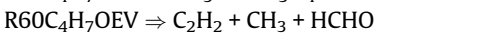 & $2.00 \times 10^{+13}$ & 0.0 & 33,000 & $\mathrm{~g}$ & 74 \\
\hline $\mathrm{R}_{62} \mathrm{C}_{4} \mathrm{H}_{7} \mathrm{OEV}=\mathrm{R}_{63} \mathrm{C}_{4} \mathrm{H}_{7} \mathrm{OEZ}$ & $5.00 \times 10^{+09}$ & 1.0 & 42,640 & c & 75 \\
\hline $\mathrm{R} 2 \mathrm{C}_{4} \mathrm{H}_{7} \mathrm{OEV} \Rightarrow \mathrm{CH}_{3}+\mathrm{C}_{3} \mathrm{H}_{4} \mathrm{OKZ}$ & $8.50 \times 10^{+12}$ & 0.0 & 28,000 & c & 76 \\
\hline 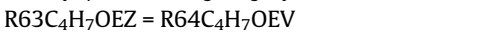 & $1.70 \times 10^{+09}$ & 1.0 & 24,540 & c & 77 \\
\hline 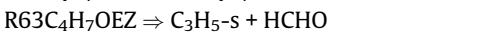 & $2.00 \times 10^{+13}$ & 0.0 & 24,000 & c & 78 \\
\hline 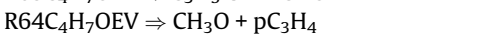 & $8.50 \times 10^{+12}$ & 0.0 & 28,000 & c & 79 \\
\hline \multicolumn{6}{|c|}{ Reactions of molecules derived from of unimolecular initiations } \\
\hline $\mathrm{CH}_{2} \mathrm{CHCH}_{2} \mathrm{OCH}_{3}=\mathrm{C}_{3} \mathrm{H}_{6}+\mathrm{CH}_{2} \mathrm{O}$ & $2.72 \times 10^{-03}$ & 4.1 & 37,212 & b & 80 \\
\hline $\mathrm{CH}_{3}+\mathrm{R}_{65} \mathrm{C}_{3} \mathrm{H}_{5} \mathrm{O}=\mathrm{CH}_{2} \mathrm{CHCH}_{2} \mathrm{OCH}_{3}$ & $1.21 \times 10^{+13}$ & 0.0 & 0.0 & b & 81 \\
\hline $\mathrm{CH}_{3} \mathrm{O}+\mathrm{C}_{3} \mathrm{H}_{5}-\mathrm{Y}=\mathrm{CH}_{2} \mathrm{CHCH}_{2} \mathrm{OCH}_{3}$ & $1.51 \times 10^{+13}$ & 0.0 & 0.0 & b & 82 \\
\hline 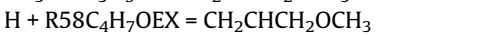 & $1.99 \times 10^{+14}$ & 0.0 & 0.0 & $\mathrm{~b}$ & 83 \\
\hline $\mathrm{CH}_{2} \mathrm{CHOCH}_{2} \mathrm{CH}_{3}=\mathrm{CH}_{3} \mathrm{CHO}+\mathrm{C}_{2} \mathrm{H}_{4}$ & $4.56 \times 10^{+01}$ & 3.0 & 41,366 & b & 84 \\
\hline $\mathrm{CH}_{3}+\mathrm{R}_{66 C} \mathrm{C}_{3} \mathrm{H}_{5} \mathrm{OET}=\mathrm{CH}_{2} \mathrm{CHOCH}_{2} \mathrm{CH}_{3}$ & $1.21 \times 10^{+13}$ & 0.0 & 0.0 & $\mathrm{~b}$ & 85 \\
\hline $\mathrm{C}_{2} \mathrm{H}_{5}+\mathrm{CH}_{2} \mathrm{CHO}=\mathrm{CH}_{2} \mathrm{CHOCH}_{2} \mathrm{CH}_{3}$ & $1.12 \times 10^{+12}$ & 0.0 & 0.0 & b & 86 \\
\hline $\mathrm{C}_{2} \mathrm{H}_{5} \mathrm{O}+\mathrm{C}_{2} \mathrm{H}_{3}=\mathrm{CH}_{2} \mathrm{CHOCH}_{2} \mathrm{CH}_{3}$ & $1.12 \times 10^{+12}$ & 0.0 & 0.0 & b & 87 \\
\hline $\mathrm{CH}_{2} \mathrm{CHCH}_{2} \mathrm{CH}_{2} \mathrm{OH}=\mathrm{C}_{3} \mathrm{H}_{6}+\mathrm{CH}_{2} \mathrm{O}$ & $8.22 \times 10^{-01}$ & 3.3 & 33,647 & $\mathrm{~b}$ & 88 \\
\hline $\mathrm{OH}+\mathrm{C}_{4} \mathrm{H}_{7}=\mathrm{CH}_{2} \mathrm{CHCH}_{2} \mathrm{CH}_{2} \mathrm{OH}$ & $2.41 \times 10^{+13}$ & 0.0 & 0.0 & $\mathrm{~b}$ & 89 \\
\hline $\mathrm{C}_{3} \mathrm{H}_{5}-\mathrm{Y}+\mathrm{CH} 2 \mathrm{OH}=\mathrm{CH}_{2} \mathrm{CHCH}_{2} \mathrm{CH}_{2} \mathrm{OH}$ & $9.64 \times 10^{+12}$ & 0.0 & 0.0 & $\mathrm{~b}$ & 90 \\
\hline 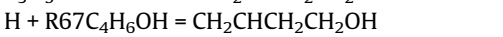 & $1.99 \times 10^{+14}$ & 0.0 & 0.0 & b & 91 \\
\hline $\mathrm{CH}_{2} \mathrm{CHCH}_{2} \mathrm{OCH}_{3}+\mathrm{H}=\mathrm{R}_{52} \mathrm{C}_{4} \mathrm{H}_{7} \mathrm{OEZ}+\mathrm{H}_{2}$ & $7.20 \times 10^{+08}$ & 1.5 & 4976 & d & 92 \\
\hline $\mathrm{CH}_{2} \mathrm{CHCH}_{2} \mathrm{OCH}_{3}+\mathrm{O}=\mathrm{R}_{52} \mathrm{C}_{4} \mathrm{H}_{7} \mathrm{OEZ}+\mathrm{OH}$ & $5.10 \times 10^{+08}$ & 1.5 & 2920 & d & 93 \\
\hline $\mathrm{CH}_{2} \mathrm{CHCH}_{2} \mathrm{OCH}_{3}+\mathrm{OH}=\mathrm{R}_{52} \mathrm{C}_{4} \mathrm{H}_{7} \mathrm{OEZ}+\mathrm{H}_{2} \mathrm{O}$ & $3.60 \times 10^{+06}$ & 2.0 & -913 & d & 94 \\
\hline 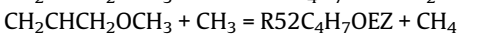 & $2.40 \times 10^{+06}$ & 1.87 & 8496 & d & 95 \\
\hline $\mathrm{CH}_{2} \mathrm{CHCH}_{2} \mathrm{OCH}_{3}+\mathrm{OOH}=\mathrm{R} 52 \mathrm{C}_{4} \mathrm{H}_{7} \mathrm{OEZ}+\mathrm{H}_{2} \mathrm{O}_{2}$ & $4.20 \times 10^{+04}$ & 2.69 & 15,923 & d & 96 \\
\hline $\mathrm{CH}_{2} \mathrm{CHCH}_{2} \mathrm{OCH}_{3}+\mathrm{H}=\mathrm{R}_{58 C_{4}} \mathrm{H}_{7} \mathrm{OEX}+\mathrm{H}_{2}$ & $5.40 \times 10^{+08}$ & 2.5 & -1900 & c & 97 \\
\hline $\mathrm{CH}_{2} \mathrm{CHCH}_{2} \mathrm{OCH}_{3}+\mathrm{O}=\mathrm{R}_{58 C_{4}} \mathrm{H}_{7} \mathrm{OEX}+\mathrm{OH}$ & $8.80 \times 10^{+10}$ & 0.7 & 3250 & c & 98 \\
\hline $\mathrm{CH}_{2} \mathrm{CHCH}_{2} \mathrm{OCH}_{3}+\mathrm{OH}=\mathrm{R} 58 \mathrm{C}_{4} \mathrm{H}_{7} \mathrm{OEX}+\mathrm{H}_{2} \mathrm{O}$ & $3.00 \times 10^{+06}$ & 2.0 & -1520 & c & 99 \\
\hline 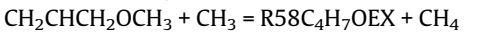 & $1.00 \times 10^{+11}$ & 0.0 & 7300 & c & 100 \\
\hline 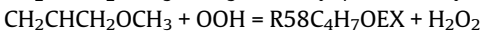 & $6.40 \times 10^{+03}$ & 2.6 & 12,400 & c & 101 \\
\hline 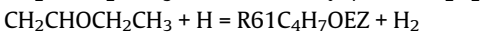 & $4.80 \times 10^{+08}$ & 1.5 & 3357 & d & 102 \\
\hline $\mathrm{CH}_{2} \mathrm{CHOCH}_{2} \mathrm{CH}_{3}+\mathrm{O}=\mathrm{R}_{1} \mathrm{C}_{4} \mathrm{H}_{7} \mathrm{OEZ}+\mathrm{OH}$ & $3.40 \times 10^{+08}$ & 1.5 & 1051 & d & 103 \\
\hline 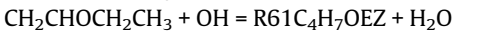 & $2.40 \times 10^{+06}$ & 2.0 & -2159 & d & 104 \\
\hline 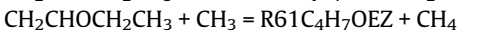 & $1.60 \times 10^{+06}$ & 1.87 & 6876 & d & 105 \\
\hline $\mathrm{CH}_{2} \mathrm{CHOCH}_{2} \mathrm{CH}_{3}+\mathrm{OOH}=\mathrm{R}_{61 \mathrm{C}_{4}} \mathrm{H}_{7} \mathrm{OEZ}+\mathrm{H}_{2} \mathrm{O}_{2}$ & $2.80 \times 10^{+04}$ & 2.69 & 14,427 & d & 106 \\
\hline 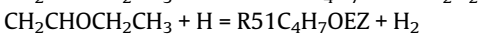 & $7.20 \times 10^{+08}$ & 1.5 & 9059 & d & 107 \\
\hline $\mathrm{CH}_{2} \mathrm{CHOCH}_{2} \mathrm{CH}_{3}+\mathrm{O}=\mathrm{R}_{5} 1 \mathrm{C}_{4} \mathrm{H}_{7} \mathrm{OEZ}+\mathrm{OH}$ & $5.10 \times 10^{+08}$ & 1.5 & 7631 & d & 108 \\
\hline 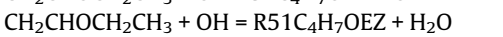 & $3.60 \times 10^{+06}$ & 2.0 & 2227 & d & 109 \\
\hline 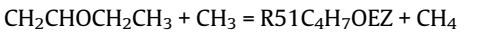 & $2.40 \times 10^{+06}$ & 1.87 & 12,579 & d & 110 \\
\hline 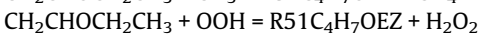 & $4.20 \times 10^{+04}$ & 2.69 & 19,692 & d & 111 \\
\hline 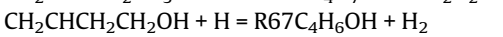 & $5.40 \times 10^{+04}$ & 2.5 & -1900 & c & 112 \\
\hline 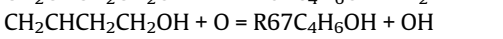 & $8.80 \times 10^{+10}$ & 0.7 & 3250 & c & 113 \\
\hline 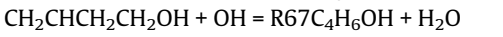 & $3.00 \times 10^{+06}$ & 2.0 & -1520 & c & 114 \\
\hline 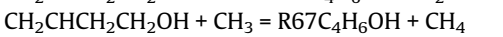 & $1.00 \times 10^{+11}$ & 0.0 & 7300 & c & 115 \\
\hline 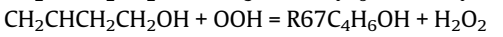 & $6.40 \times 10^{+03}$ & 2.6 & 12,400 & c & 116 \\
\hline 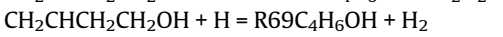 & $4.80 \times 10^{+08}$ & 1.5 & 3347 & d & 117 \\
\hline 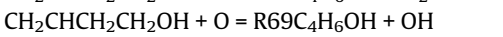 & $3.40 \times 10^{+08}$ & 1.5 & 1040 & d & 118 \\
\hline 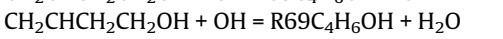 & $2.40 \times 10^{+06}$ & 2.0 & -2166 & d & 119 \\
\hline 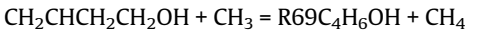 & $1.60 \times 10^{+06}$ & 1.87 & 6866 & d & 120 \\
\hline 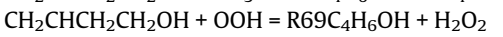 & $2.80 \times 10^{+04}$ & 2.69 & 14,419 & d & 121 \\
\hline $\mathrm{C}_{2} \mathrm{H}_{3}+\mathrm{CH}_{2} \mathrm{CHO}=\mathrm{CH}_{2} \mathrm{CHCH}_{2} \mathrm{CHO}$ & $1.10 \times 10^{+12}$ & 0.0 & 0.0 & $\mathrm{~b}$ & 122 \\
\hline
\end{tabular}


Table 3 (continued)

\begin{tabular}{|c|c|c|c|c|c|}
\hline Reactions & $A$ & $n$ & $E_{a}$ & Footnote & $\mathrm{n}^{\circ}$ \\
\hline $\mathrm{C}_{3} \mathrm{H}_{5}-\mathrm{Y}+\mathrm{HCO}=\mathrm{CH}_{2} \mathrm{CHCH}_{2} \mathrm{CHO}$ & $1.10 \times 10^{+12}$ & 0.0 & 0.0 & b & 123 \\
\hline $\mathrm{C}_{2} \mathrm{H}_{3}+\mathrm{CH}_{2} \mathrm{CHO}=\mathrm{CH}_{2} \mathrm{CHOCHCH}_{2}$ & $1.10 \times 10^{+12}$ & 0.0 & 0.0 & b & 124 \\
\hline 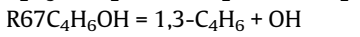 & $2.00 \times 10^{+13}$ & 0.0 & 26,000 & b & 125 \\
\hline $\mathrm{R}_{69} \mathrm{C}_{4} \mathrm{H}_{6} \mathrm{OH}=\mathrm{C}_{2} \mathrm{H}_{3} \mathrm{OH}+\mathrm{C}_{2} \mathrm{H}_{3}$ & $2.00 \times 10^{+13}$ & 0.0 & 35,500 & b & 126 \\
\hline 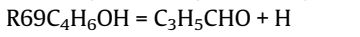 & $2.50 \times 10^{+13}$ & 0.0 & 29,000 & b & 127 \\
\hline
\end{tabular}

a Rate constant taken equal to that of the recombination of $\mathrm{H}$ atoms with alkyl radicals as proposed by Allara and Shaw [38].

b Directly taken from the work on the high-temperature pyrolysis of THF by Verdicchio et al. [16].

c Directly provided by EXGAS software [36].

d Estimated using the Evans-Polanyi correlation proposed by Dean and Bozelli [39].

e Calculated using the quantum methods described in the text.

${ }^{\mathrm{f}}$ Calculated using the quantum methods described in the text, with $\mathrm{A}$ factor divided by 2 to improve agreement with experimental results.

g Rate constant taken equal to that of $\mathrm{CHCHCH}_{2} \mathrm{CH}_{2} \mathrm{CH}_{3} \Rightarrow \mathrm{C}_{3} \mathrm{H}_{7}+\mathrm{C}_{2} \mathrm{H}_{2}$ as provided by EXGAS software.

at the same place along the axis of the tube as the last pressure transducer. The ignition delay time was defined as the time interval between the pressure rise measured by the last pressure transducer, due to the arrival of the reflected shock wave, and the rise of the optical signal by the photomultiplier up to $10 \%$ of its maximum value (the time resolution of the emission measurements is better than $2 \mu \mathrm{s}$ ). Fresh reaction mixtures were prepared every day. Before each introduction of the reaction mixture, the reaction section was flushed with pure argon and evacuated, for insuring the residual gas to be mainly argon.

This study was performed under the following experimental conditions, after the reflected shock wave:

- Temperature range from 1300 to $1700 \mathrm{~K}$ and pressure range from 811 to $932 \mathrm{kPa}$.

- Mixtures (argon/THF/oxygen, in molar percent) were (94/0.5/ 5.5), (98.375/0.25/1.375), (96.75/0.5/2.75), (93.5/1.0/5.5), and $(98.125 / 0.5 / 1.375)$ corresponding to three different equivalence ratios ( $\varphi=0.5,1$ and 2$)$ and three initial THF mole fractions $(0.25,0.5$, and 1$)$.

\subsection{Simulation methods}

The kinetic model of THF combustion described in the next section, especially in Table 3, was used for simulations without any change. Simulations were performed using softwares from CHEMKIN package [33]. Flame simulations were performed using the PREMIX software. For flame structures, convergence criteria were decreased until a grid independent solution was found (GRAD and CURV of 0.5). To compensate for the perturbations induced by sampling quartz probe and thermocouple, the temperature profile used in simulation is an average between the experimental profiles measured with and without the quartz probe, shifted $1.1 \mathrm{~mm}(\varphi=1.0), 1.2 \mathrm{~mm}(\varphi=1.3)$, and $1.4 \mathrm{~mm}(\varphi=0.7)$ away from the burner surface. Simulations of adiabatic laminar burning velocities were carried out with a GRAD and CURV of 0.05 and include thermal diffusion effect. Shock tube simulations were performed using SENKIN software. Computed ignition delay times were determined from the $10 \%$ rise of $\mathrm{OH}^{*}$ radical mole fraction, with the same mechanism considered for this excited species as in the work of Sirjean et al. [34]. Note that the experimental $\mathrm{OH}$ emission at $306 \mathrm{~nm}$ is related to electronically excited $\mathrm{OH}^{*}$ concentration and is not directly proportional to $\mathrm{OH}$ radical concentration.

\section{Kinetic model development}

A new detailed chemical kinetic model for the high-temperature combustion of THF was developed using the automatic generator EXGAS software [31,35-37] with a $C_{0}-C_{6}$ reaction database.
Then, some key kinetic parameters were updated to take into account the specificity of cyclic ethers combustion chemistry. Table 3 presents the reactions in the THF primary mechanism. The detailed structures of large species involved in Table 3 can be found in Table S1 in the Supplemental Material. The complete mechanism includes 255 species in 1723 reactions and it is available, together with the thermochemical and transport data file, in Supplemental Material under CHEMKIN format.

\subsection{Main features of the mechanism}

Like all the mechanisms generated by EXGAS, the present one basically consists of three parts [35]:

- The first part is a reaction base that contains the reactions of the species for which the automatic generation is difficult. In most work based on EXGAS, a $C_{0}-C_{2}$ reaction base was used for species including two carbon atoms or less [36]. Here a new version of the software [40] has allowed the direct inclusion in the mechanism of a $C_{0}-C_{6}$ reactions base, consisting of the $C_{0}-C_{2}$ reaction base, but also of reactions of $C_{3}-C_{6}$ unsaturated species, such propene or butadienes [41], validated using experiments produced mostly using the low-pressure flame device described here-before, as well as of reactions of light aromatic compounds up to ethylbenzene [42]. Compared to this reference, the submechanism for ethenol reactions, taken from our ethanol kinetic model [24] has been added. Reactions consuming cyclopropanecarboxaldehyde, butanal and 2-butenal have been written in more details, these last ones being mainly taken from [43]. Reactions involving fulvene and leading to benzene formation have been added, as proposed by [44].

- The second part is a comprehensive primary mechanism containing the reactions of the reactant and of the radicals deriving from it. In the case of THF high-temperature oxidation, these include unimolecular/bimolecular initiation steps (except the unimolecular initiations specific the cyclic species which have to be treated separately as shown further in this text), as well as beta-scission, isomerisations, and metathesis reactions. The kinetic values automatically provided by EXGAS are those usually used in the case of acyclic alkanes [36] and alkenes [37], since ring opening reactions yield unsaturated species. Due to the presence of the O-atom in the THF cyclic molecule, these values issued from structure reactivity relationships had to be adapted. The kinetic values automatically generated have been used without changes only in the case of the unimolecular initiations involving the breaking of a $\mathrm{C}-\mathrm{H}$ bond (reactions 1 and 2 in Table 3) and the bimolecular initiations of THF with $\mathrm{O}_{2}$ leading to tetrahydrofur-2-yl (THF-yl-2) and tetrahydrofur-3-yl (THF-yl-3) radicals (reactions 19 and 20). EXGAS generated kinetic data are also used for some beta-scissions of linear 
radicals produced from THF-yl-2 (reactions 51, 53-57) or THFyl-3 (reactions 71-73 and 75-79) by a ring-opening followed by an isomerization, the isomerizations of the resonance stabilized radical produced from THF-yl-3 by ring-opening (reactions 63-65) and the $\mathrm{H}$-abstractions by $\mathrm{CHO}, \mathrm{CH}_{2} \mathrm{OH}, \mathrm{CH}_{3} \mathrm{O}, \mathrm{CH}_{3} \mathrm{OO}$ and $\mathrm{C}_{2} \mathrm{H}_{5}$ radicals (reactions 31-40). For the other reactions, as described below, rate constants based on quantum calculation or on Evans-Polanyi correlations have been used.

- The third one is a global secondary mechanism that contains the reactions of the molecular products formed in the primary mechanism. However this part has not been taken into account here, since the molecular products formed in the primary mechanism are either species the reactions of which are already included in the $\mathrm{C}_{0}-\mathrm{C}_{6}$ reactions base, or cyclic molecules that cannot be treated by EXGAS, but whose reaction are part of the mechanism proposed for the combustion of furan in our recent study [25]. This last submechanism has been added to the present mechanism.

Thermochemical data for molecules or radicals were automatically calculated and stored as 14 polynomial coefficients, these are calculated using the software THERGAS [45] based on the group and bond additivity methods proposed by Benson [46]. The transport properties of the species were evaluated by using an in-house code for species for which no data were available (mainly for species not included in the $\mathrm{C}_{0}-\mathrm{C}_{2}$ reaction base).

\subsection{Methods used for theoretical rate constant calculations}

In this work, the kinetic data of several $\mathrm{H}$-abstractions and beta-scissions have been obtained from theoretical calculations. Potential energy surfaces (PES) were computed at the CBS-QB3 level of theory, using Gaussian 09 software [47]. The rate coefficients were determined using the MULTIWELL 2013 suite of codes [48]. High-pressure limit rate coefficients were obtained from canonical transition state theory (Eq. (1)):

$k_{\infty}(T)=\kappa(T) L \frac{k_{B} T}{h} \frac{Q_{\mathrm{TS}}(T)}{Q_{R}(T)} \exp \left[-\frac{V^{\ddagger}}{R T}\right]$,

where $V^{\neq}$is the classical barrier height; $Q_{\mathrm{TS}}(T)$ and $Q_{R}(T)$ represent the partition functions calculated, respectively, for the transition state (TS) and the reactant $(R) ; \kappa(T)$ is the transmission coefficient; and $L$ is the statistical factor defined as:

$L=\frac{\sigma_{R} \times n_{\mathrm{TS}}}{\sigma_{\mathrm{TS}} \times n_{R}}$

In Eq. (2), $\sigma_{R}$ and $\sigma_{\mathrm{Ts}}$ are the external symmetry of, respectively, the reactant and the transition state, while $n_{R}$ and $n_{\mathrm{TS}}$ correspond to the number of optical isomers for reactant and transition state, respectively. The vibrational partition function was calculated using scaled harmonic frequencies, except for internal rotations, which were treated as hindered rotations (HR) by means of the 1D-HR approximation [49]. Relaxed scans of each hindered rotor have been performed at the B3LYP/cbsb7 level of theory and the resulting potential energy fitted with a Fourier series of the type shown in Eq. (3):

$\mathrm{V}(\theta)=V_{0}+\sum_{n=1}^{5} V_{n}^{c} \cos \left(n \sigma_{v} \theta\right)+\sum_{n=1}^{5} V_{n}^{s} \sin \left(n \sigma_{v} \theta\right)$

where $\theta$ is the scanned dihedral angle in radians, $\sigma_{\nu}$ is the symmetry number for the potential energy and $V_{n}^{c}, V_{n}^{s}$ and $V_{0}$ the 11 fitted parameters. The inertia of each rotor has been considered constant along the entire scan and its value calculated with the CHEMRATE software [50] which was found to yield more accurate results,
Table 4

Ab initio calculated thermochemical parameters for $T=298.15 \mathrm{~K}$ for species of Table 3.

\begin{tabular}{lcll}
\hline Species & $\Delta \mathrm{H}_{\mathrm{f}}{ }^{\circ}(\mathrm{kcal} / \mathrm{mol})$ & $\mathrm{S}^{\circ}(\mathrm{cal} / \mathrm{K} / \mathrm{mole})$ & $\mathrm{C}_{\mathrm{p}}{ }^{\circ}(\mathrm{cal} / \mathrm{K} / \mathrm{mol})$ \\
\hline THF & -44.06 & 72.43 & 19.18 \\
THF-yl-2 & -2.23 & 73.09 & 19.51 \\
THF-yl-3 & 2.12 & 74.03 & 19.95 \\
$\mathrm{R}_{0} \mathrm{C}_{4} \mathrm{H}_{7} \mathrm{OA}$ & 0.66 & 86.68 & 25.04 \\
$\mathrm{R}_{1} \mathrm{C}_{4} \mathrm{H}_{7} \mathrm{OEZ}$ & 17.86 & 84.09 & 26.15 \\
$\mathrm{R}_{33 C_{4} \mathrm{H}_{7} \mathrm{OZ}}$ & 18.62 & 82.23 & 24.45 \\
$\mathrm{R}_{2} \mathrm{C}_{4} \mathrm{H}_{7} \mathrm{OEZ}$ & 19.55 & 85.87 & 23.62 \\
$\mathrm{R} 55 \mathrm{C}_{4} \mathrm{H}_{7} \mathrm{OK}$ & -11.70 & 84.63 & 22.95 \\
$\mathrm{R} 54 \mathrm{C}_{4} \mathrm{H}_{7} \mathrm{OA}$ & -11.26 & 80.69 & 22.42 \\
$2,3-\mathrm{DHF}$ & -18.16 & 70.85 & 17.79 \\
2,5-DHF & -15.22 & 67.97 & 17.73 \\
\hline
\end{tabular}

because of the method used for the definition of the rotation axis, than the MOMINERT module of MULTIWELL [48].

Quantum tunneling was taken into account using the Eckart [51] approximation and a three parameters Arrhenius expression fitted to the computed values between 200 and $3000 \mathrm{~K}$.

In order to keep consistency with the kinetic data, thermochemical parameters of the involved species have been recalculated at different temperatures using the THERMO module of MULTIWELL package [48]. In Table 4 enthalpy of formation $\left(\Delta \mathrm{H}_{\mathrm{f}}{ }^{\circ}\right)$, entropy $\left(\mathrm{S}^{\circ}\right)$ and heat capacity at constant pressure $\left(\mathrm{C}_{\mathrm{p}}{ }^{\circ}\right)$ of important species are reported for $T=298.15 \mathrm{~K}$. The calculated values were then fitted to the NASA polynomials format in order to be included in the CHEMKIN mechanism.

\subsection{Description of the primary mechanism}

In this section, the key elements of the primary mechanism, given in Table 3, are discussed in detail. The structure of the THF molecule is compared to that of furan in Fig. 1. In the THF molecule, the calculated bond energy of ring $\mathrm{C} 2,5-\mathrm{H}$ and $\mathrm{C} 3,4-\mathrm{H}$ bonds are 93.9 and $98.3 \mathrm{kcal} \mathrm{mol}^{-1}$, respectively, which are in very good agreement with those calculated by Simmie [2]. These bonds are much weaker than those of furan (119.3-119.5 kcal mol ${ }^{-1}$ [52]). In the THF molecule, the closeness of the $O$ atom weakens the $\mathrm{C}-\mathrm{H}$ bond, while it is not significantly affected in the furan aromatic molecule.

\subsubsection{The unimolecular initiations of THF}

A detailed theoretical study of the unimolecular initiations of THF has been performed by Verdicchio et al. [16] for their model of the pyrolysis of this molecule. Five types of unimolecular initiations pathways have been taken in account for THF (reactions 1-9): (a) initial $\mathrm{C}-\mathrm{H}$ bond fissions in the THF-ring, (b) ring opening by $\mathrm{C}-\mathrm{O}$ and $\mathrm{C}-\mathrm{C}$ bond fission reactions yielding molecular products or radicals via diradical species, (c) a pericyclic rearrangement to yield 1-buten-4-ol $\left(\mathrm{CH}_{2} \mathrm{CHCH}_{2} \mathrm{CH}_{2} \mathrm{OH}\right)$, (d) ring opening by $\mathrm{C}-\mathrm{O}$ and $\mathrm{C}-\mathrm{C}$ bond fission with internal hydrogen atom transfer leading to the formation of carbenic intermediates that isomerize and decompose into molecular products, and (e) a pericyclic hydrogen elimination involving the formation of cyclic diradical. A scheme presenting pathways ( $\mathrm{b}$ to e) is given in Fig. S1 in the Supplemental Material. A master equation modeling was used to determined pressure dependent rate coefficients. Reactions involving diradicals and carbenes are formally direct reactions yielding directly products. For reactions 3-9, pressure effects have been considered and the rate coefficients which have been used, are those calculated at $0.1,1$ and 10 atm by Verdicchio et al. [16]. The acyclic molecules (butanal, as well as $\mathrm{C}_{4}$ unsaturated ethers and enols (see reactions $80-127$ in Table 3 ) for the two last types of species) react either by retro-ene reactions (reactions $80,84,88$ ) [16], by 
unimolecular initiations [16], or by $\mathrm{H}$-abstractions with $\mathrm{H}, \mathrm{O}, \mathrm{OH}$, $\mathrm{CH}_{3}$ and $\mathrm{HO}_{2}$ radicals with, in this last case, rate constants estimated from the Evans-Polanyi correlation proposed by Dean and Bozelli [39] or directly taken from EXGAS.

\subsection{2. $H$-abstractions from $T H F$ and derived reactions}

Because of the symmetry of THF, only two types of hydrogen atoms can be considered in this reaction class: $\mathrm{H}$-abstractions from the carbon atoms (atoms 2 and 5, in Fig. 1) directly bonded to oxygen (producing the THF-yl-2 radical) and from the other secondary carbon atoms (atoms 4 and 3, leading to THF-yl-3 radical).

The rate parameters for $\mathrm{H}$-abstractions by $\mathrm{H}$ and $\mathrm{CH}_{3}$ radicals (reactions 23, 24, 29 and 30) were obtained from transition state theory calculations, with barrier heights calculated at the CBSQB3 level of theory (see values in Table S2 in Supplementary Material), whereas rate coefficients for $\mathrm{H}$-abstractions by $\mathrm{O}$ (reactions 21 and 22), $\mathrm{OH}$ (reactions 25 and 26) and $\mathrm{HO}_{2}$ (reactions 27 and 28) were deduced from the Evans-Polanyi correlation proposed by Dean and Bozelli [39].

Since the decomposition of the THF-yl-2 and THF-yl-3 radicals is the central piece of the characterization of the THF high-temperature oxidation, this set of reactions has also been described with theoretical calculations. Similar isomerizations and decomposition pathways, as proposed in the work on 2,5-dimethyltetrahydrofuran by Simmie [2], have been taken into account for THF. The related PES have been calculated at the CBS-QB3 level of theory. The related rate constants have been computed at their high pressure limit. Note that further more accurate determinations of the kinetic data of these reactions accounting for pressure effects, which could be have some influence especially under low-pressure flame conditions, would require using a rigorous master-equation treatment. Kinetic parameters for the THF-yl-2 to THF-yl-3 interconversion reaction by internal $\mathrm{H}$-atom transfer (reaction 41), has been also computed and included in the model. Our calculations show a barrier of $45 \mathrm{kcal} / \mathrm{mol}$ going from THF-yl-2 to THFyl-3 and of $41 \mathrm{kcal} / \mathrm{mol}$ for the reverse reaction.

The PES, for all the investigated decomposition reactions, are presented in Figs. 2 and 3 for THF-yl-2 and THF-yl-3 radicals, respectively. Figure S2 in the Supplemental Material shows the high-pressure limit temperature-dependent branching ratios for the unimolecular decompositions of THF-yl-2 and THF-yl-3 radicals. As show in Fig. 2, the ring in THF-yl-2 radical can open by $\beta$-scission of $\mathrm{C}-\mathrm{O}$ and $\mathrm{C}-\mathrm{C}$ bonds to form $\mathrm{R}^{2} \mathrm{C}_{4} \mathrm{H}_{7} \mathrm{OA}$ (reaction

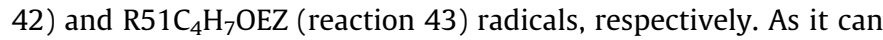
be expected, the $\mathrm{C}-\mathrm{O}$ bond scission is energetically favored, with a barrier about $11 \mathrm{kcal} / \mathrm{mol}$ lower than the $\mathrm{C}-\mathrm{C}$ bond scission. As shown in Fig. S2a, THF-2-yl unimolecular decomposition proceeds completely through the $\mathrm{C}-\mathrm{O}$ bond-scission reaction (formation of

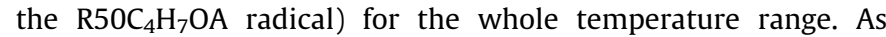
already discussed above, this reaction shows the lowest activation energy and is therefore the energetically favored pathway. Isomerization reactions by internal $\mathrm{H}$-atom transfer can occur from

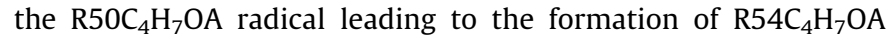
(reaction 45) and $\mathrm{R}_{55} \mathrm{C}_{4} \mathrm{H}_{7} \mathrm{OK}$ (reaction 46) radicals. Because of the low barrier showed by this last isomerization, $\alpha$-scission of

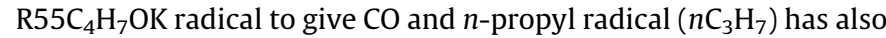

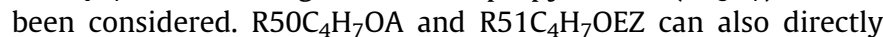
decompose by $\beta$-scission to give $\mathrm{C}_{2} \mathrm{H}_{4}$ and the $\mathrm{CH}_{2} \mathrm{CHO}$ radical, (reactions 47 and 50 respectively). The combination of THF-yl-2 radical with methyl radical to give MTHF has also been taken into account (reaction 58).

A similar pattern can be applied for the THF-yl-3 radical. In this case, the ring-opening reactions (reactions 59 and 60) lead to the formation of an oxygen-centered radical $\left(\mathrm{R}_{53} \mathrm{C}_{4} \mathrm{H}_{7} \mathrm{OZ}\right)$ and a carbon centered radical $\left(\mathrm{R}_{52} \mathrm{C}_{4} \mathrm{H}_{7} \mathrm{OEZ}\right)$, which can undergo

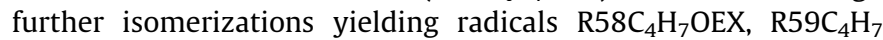

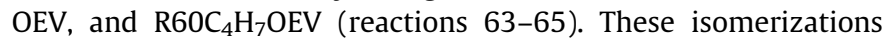
are not shown in Fig. 3 and were not theoretically treated. Both $\mathrm{R}_{52} \mathrm{C}_{4} \mathrm{H}_{7} \mathrm{OEZ}$ and $\mathrm{R}_{53} \mathrm{C}_{4} \mathrm{H}_{7} \mathrm{OZ}$ can undergo further $\beta$-scissions to give allyl radical $\left(\mathrm{C}_{3} \mathrm{H}_{5}-\mathrm{Y}\right)$ and formaldehyde (reactions 66 and 67).

Elimination of a hydrogen atom can also occur from THF-yl-2 and THF-yl-3 radicals leading to the formation of 2,3-dihydrofuran (2,3-DHF) or 2,5-dihydrofuran (2,5-DHF). As depicted in Fig. 2, the THF-yl-2 radical can only lead to 2,3-DHF with a very high barrier that can unlikely compete with the ring opening reactions. The THF-yl-3 radical, on the other hand, can lead to the formation of both 2,3-DHF and 2,5-DHF, as reported in Fig. 3. The involved reaction barriers suggest that the process is competitive with the $\beta$ scissions that lead to ring opening. These reactions are included

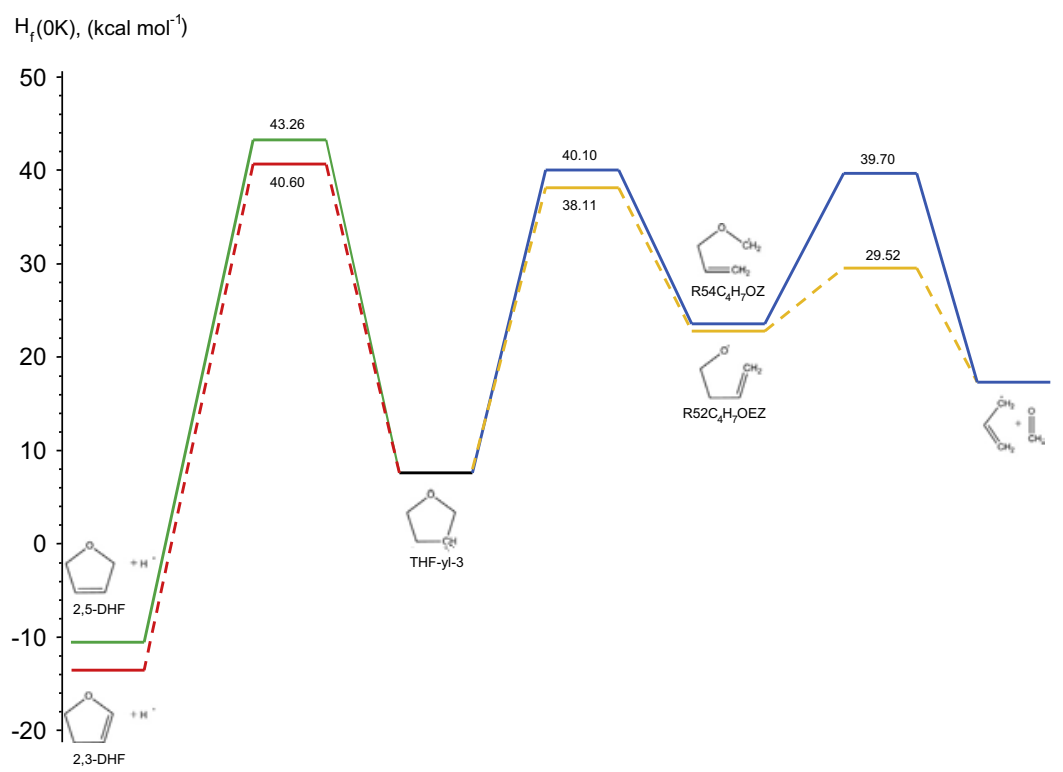

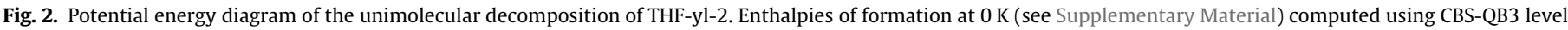
of theory. Enthalpy of formation at $0 \mathrm{~K}$ in $\mathrm{kcal} / \mathrm{mol}$ have been reported for each transition state. 


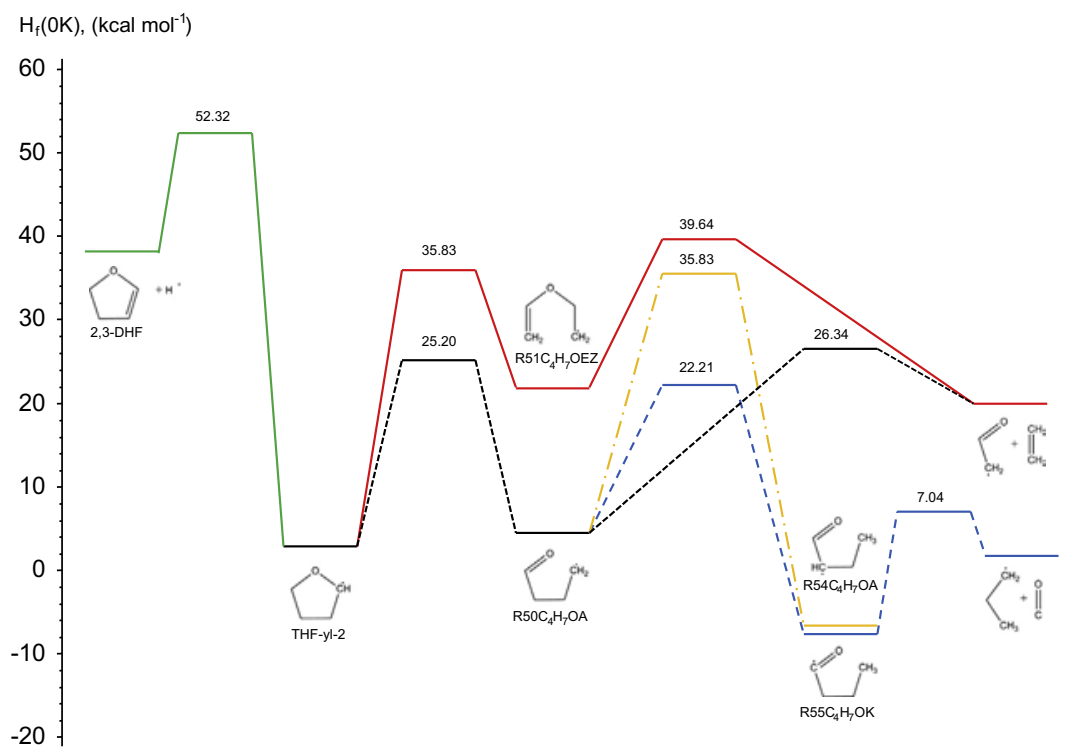

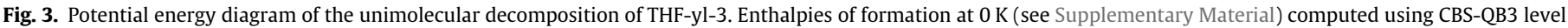
of theory. Enthalpy of formation at $0 \mathrm{~K}$ in $\mathrm{kcal} / \mathrm{mol}$ have been reported for each transition state.

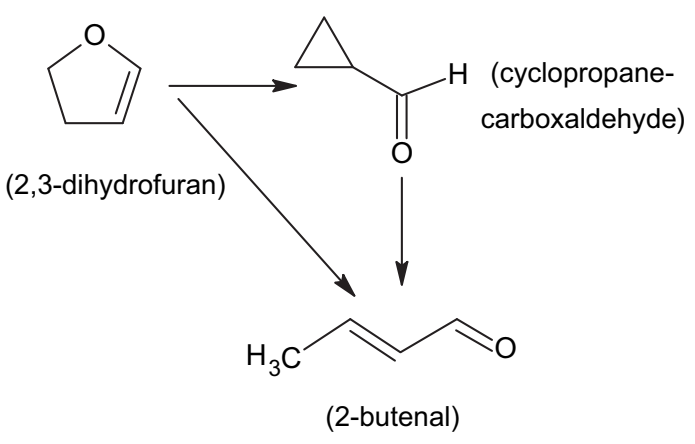

Fig. 4. Formation of cyclopropanecarboxaldehyde and 2-butenal from 2,3-dihydrofuran $[53,54]$.

in the mechanism (reactions 44, 61 and 62). Unimolecular decomposition reactions for the THF-3-yl show comparable barriers and the kinetic is therefore driven mostly by entropy. As depicted in Fig. S2b at $800 \mathrm{~K}$ THF-3-yl decompose for about $60 \%$ to the $\mathrm{R}^{2} \mathrm{C}_{4}$ $\mathrm{H}_{7} \mathrm{OA}$ radical and for about $20 \%$ to $2,3-\mathrm{DHF}$ and to $\mathrm{R}_{54} \mathrm{C}_{4} \mathrm{H}_{7} \mathrm{OA}$ radical. As temperature increases, the yields of two dihydrofurans increase and become higher than the $\mathrm{C}-\mathrm{C}$ and $\mathrm{C}-\mathrm{O}$ bond fission product yields for temperatures higher than $1800 \mathrm{~K}$.

The secondary reactions of 2,3-DHF and 2,5-DHF are mostly those already considered in the secondary mechanism proposed for furan by Tian et al. [52] and used by Liu et al. [25]. As discussed by the team of Lifshitz [53,54], the isomerizations of 2,3-dihydrofuran can yield 2-butenal and cyclopropanecarboxaldehyde that subsequently isomerizes to 2-butenal as is shown in Fig. 4. These reactions are considered in the present mechanism. The reactions of MTHF have been mostly taken from [43].

\section{Experimental and simulated results}

Results obtained in low-pressure and atmospheric premixed flames, and in a shock tube are presented in this section. Excel files providing tables of all these experimental results are given as Supplemental Material.

\subsection{Combustion of THF in low-pressure premixed flat flame}

The chemical structure of THF flames was investigated at three equivalences ratios ( $\varphi=0.7,1.0$ and 1.3). About 40 species were identified and quantified. In all experiments, the carbon (C), hydrogen $(\mathrm{H})$, and oxygen $(\mathrm{O})$ atom balances were checked. The difference between inlet and outlet is $\sim 3-4 \%$ for $C, \sim 5-9 \%$ for $O$ and $\mathrm{H}$. The flame temperature profiles and the mole fraction profiles of selected important species are presented and discussed in the following paragraphs.

Figures 5-12 present the temperature and mole fraction profiles of $\mathrm{C}_{0}-\mathrm{C}_{6}$ species (major and intermediate) as a function of the distance above the burner $(h)$. In all these figures, the symbols correspond to experimental results and the lines to simulated results. These figures show that the reaction zone peaks at $\sim 1-3 \mathrm{~mm}$ above the burner. The mole fraction of most non-oxygenated intermediates increases when increasing the equivalence ratio.

Figure 5 presents the temperature profiles measured for the three flames with and without the sampling probe. This figure shows that presence of the probe induces a thermal perturbation, which causes measured temperature with the probe to be lower by about $200 \mathrm{~K}$ than those without the probe. In this last case the lowest temperatures were around $1000-1100 \mathrm{~K}$ at $0.2-$ $0.3 \mathrm{~mm}$ height. Because of the size of the thermocouple, it was not possible to measure temperatures closer to the burner than this distance. Without the probe, the maximum measured temperatures were around $2160-2270 \mathrm{~K}$ in the post flame region $(h>6 \mathrm{~mm})$.

Figure 6 displays the consumption of reactants (THF and $\mathrm{O}_{2}$ ), as well as the mole fraction evolution of diluent (Ar) and main products $\left(\mathrm{CO}, \mathrm{CO}_{2}, \mathrm{H}_{2} \mathrm{O}\right.$, and $\left.\mathrm{H}_{2}\right)$. This figure shows that THF is completely consumed at a height of $2.0 \mathrm{~mm}$. A significant mole fraction of $\mathrm{O}_{2}\left(1-8 \times 10^{-2}\right)$ remains in the post flame region. The profiles of CO display marked maxima $\left(\sim 6-12 \times 10^{-2}\right)$ at a height of $2.5-3 \mathrm{~mm}$. It can be seen that there is a remaining mole fraction of $\mathrm{CO}$ in the post flame region, even in lean mixture. This figure shows that the model reproduces very well the consumption of reactants, the formation of main products, and also the diluent mole fraction evolution.

Figure 7 displays the mole fraction profiles of $\mathrm{C}_{1}-\mathrm{C}_{2}$ hydrocarbon intermediates, including methane $\left(\mathrm{CH}_{4}\right)$, acetylene $\left(\mathrm{C}_{2} \mathrm{H}_{2}\right)$, 

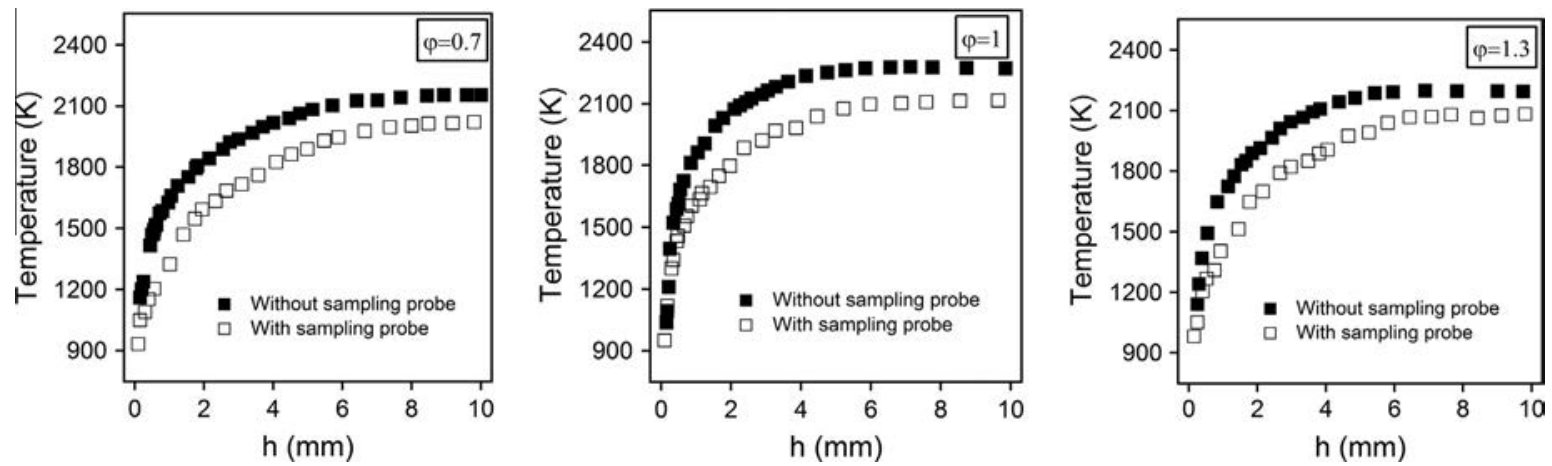

Fig. 5. Flame temperature profiles as a function of height above burner $(h)$ measured without and with the sampling probe.
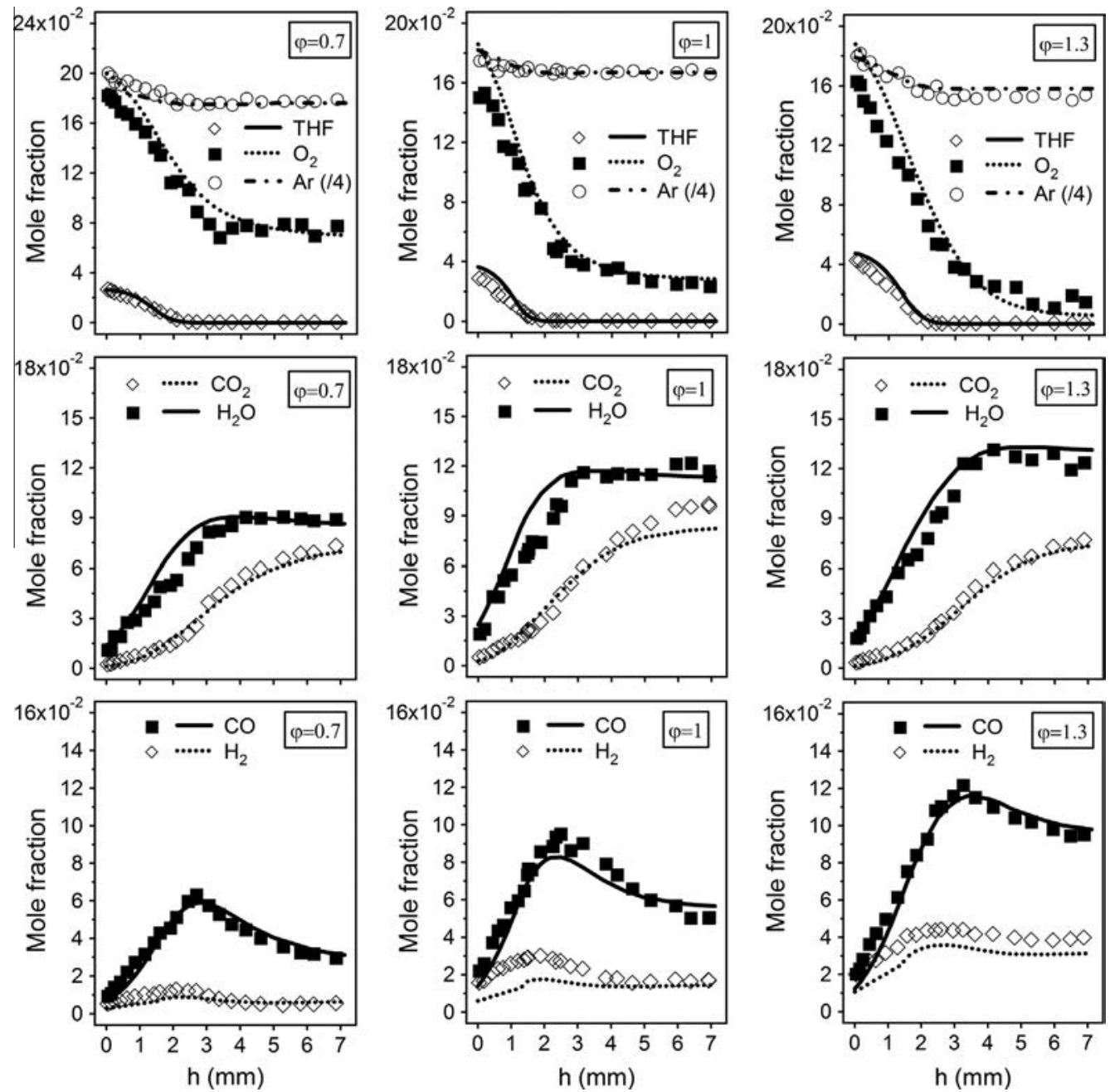

Fig. 6. Mole fraction profiles of major species (fuel, $\mathrm{O}_{2}, \mathrm{Ar}, \mathrm{CO}_{2}, \mathrm{CO}, \mathrm{H}_{2} \mathrm{O}$, and $\mathrm{H}_{2}$ ). Symbols: experiment; lines: simulation.

ethylene $\left(\mathrm{C}_{2} \mathrm{H}_{4}\right)$, and ethane $\left(\mathrm{C}_{2} \mathrm{H}_{6}\right) . \mathrm{C}_{2} \mathrm{H}_{4}$ is the most abundant intermediate among all intermediates detected in the THF flames, with maximum mole fractions of $\sim 8 \times 10^{-3}$ (at $\varphi=0.7$ ), $\sim 12 \times 10^{-3}$ (at $\varphi=1.0$ ), and $\sim 16 \times 10^{-3}$ (at $\varphi=1.3$ ), respectively. Note that for unsaturated cyclic ethers of the furan family $[25,52,55,56], \mathrm{C}_{2} \mathrm{H}_{2}$ was the most abundant intermediate. The mole fraction profiles of $\mathrm{CH}_{4}, \mathrm{C}_{2} \mathrm{H}_{2}$, and $\mathrm{C}_{2} \mathrm{H}_{6}$ reach their maxima at 1.5$2.5 \mathrm{~mm}$ above the burner, with maximum mole fractions of $2.7 \times 10^{-3}, 2.8 \times 10^{-3}$, and $1.5 \times 10^{-3}$, respectively in the stoichiometric flame. Under the same flame conditions and using the same analytical techniques, the mole fractions of $\mathrm{C}_{2} \mathrm{H}_{4}$ and $\mathrm{C}_{2} \mathrm{H}_{2}$ reach higher levels in the THF flame than in the ethanol flame reported by Tran et al. [24] (a factor of $\sim 2.5$ ). In the less diluted THF flame studied by Kasper et al. [20] $(\varphi=1.0, P=2 \mathrm{kPa}$, and dilution $28 \%$ ), the mole fractions of $\mathrm{C}_{2} \mathrm{H}_{4}, \mathrm{C}_{2} \mathrm{H}_{2}$, and $\mathrm{CH}_{4}$ were $\sim 2-3$ times larger than those in the present stoichiometric THF flame with $78 \%$ dilution. A discussion of the simulations of the data of Kasper et al. [20] using the present model is presented in paragraph here after. Figure 7 shows that the mole fraction profiles of all these $C_{1}-C_{2}$ hydrocarbon intermediates are very well predicted 

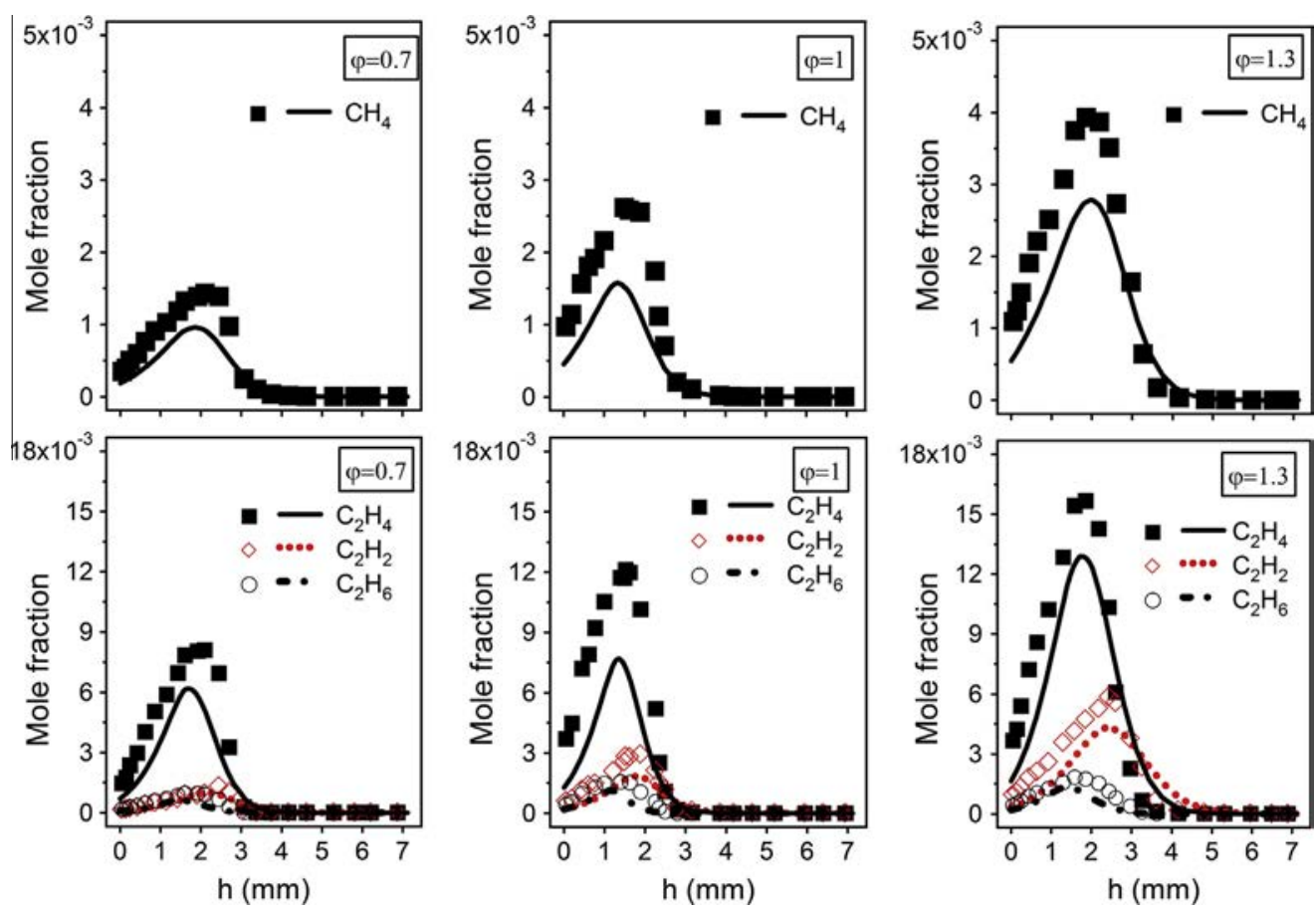

Fig. 7. Mole fraction profiles of $C_{1}-C_{2}$ hydrocarbon intermediates. Symbols: experiment; lines: simulation.
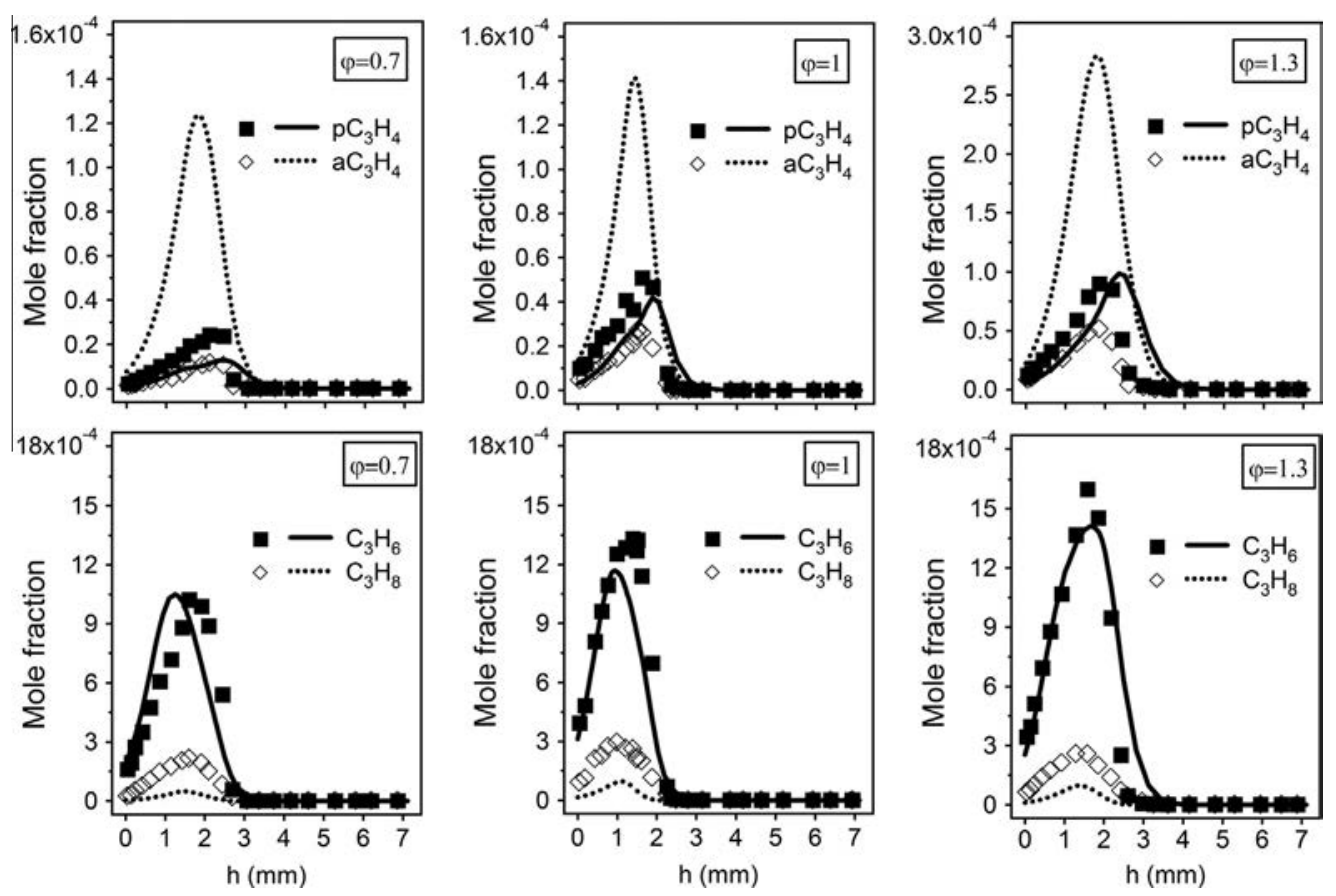

Fig. 8. Mole fraction profiles of $C_{3}$ hydrocarbon intermediates. Symbols: experiment; lines: simulation.

by the model. This applies to the profile shapes as well as to the mole fraction values.

Figure 8 displays the mole fraction profiles of $\mathrm{C}_{3}$ hydrocarbon intermediates, including two isomers of $\mathrm{C}_{3} \mathrm{H}_{4}$ (propyne $\left(\mathrm{pC}_{3} \mathrm{H}_{4}\right)$ and allene $\left.\left(\mathrm{aC}_{3} \mathrm{H}_{4}\right)\right)$, propene $\left(\mathrm{C}_{3} \mathrm{H}_{6}\right)$, and propane $\left(\mathrm{C}_{3} \mathrm{H}_{8}\right)$. The mole fraction of $\mathrm{pC}_{3} \mathrm{H}_{4}$ is larger than that of $\mathrm{aC}_{3} \mathrm{H}_{4}$ (with a factor of about 2 ). The formation of $\mathrm{C}_{3} \mathrm{H}_{8}$ does not strongly vary when increasing equivalence ratio. $\mathrm{C}_{3} \mathrm{H}_{6}$ is an abundant $\mathrm{C}_{3}$ intermediates with maximum mole fractions of $\sim 1.0 \times 10^{-3}$ (at $h=1.8 \mathrm{~mm}$ ) $\sim 1.3 \times 10^{-3}$ (at $h=1.2 \mathrm{~mm}$ ), and $\sim 1.6 \times 10^{-3}$ (at $h=1.8 \mathrm{~mm}$ ) in fuel-lean, stoichiometric, and fuel-rich flames, respectively. These mole fraction values are significantly larger than those of other $\mathrm{C}_{3}$ and heavier species. Dagaut et al. [19] and Kasper et al. [20] also found this trend in their studies. The model well reproduces the formation of this abundant species $\left(\mathrm{C}_{3} \mathrm{H}_{6}\right)$ and $\mathrm{pC}_{3} \mathrm{H}_{4}$, but predictions are less satisfactory for the other $C_{3}$ species. 

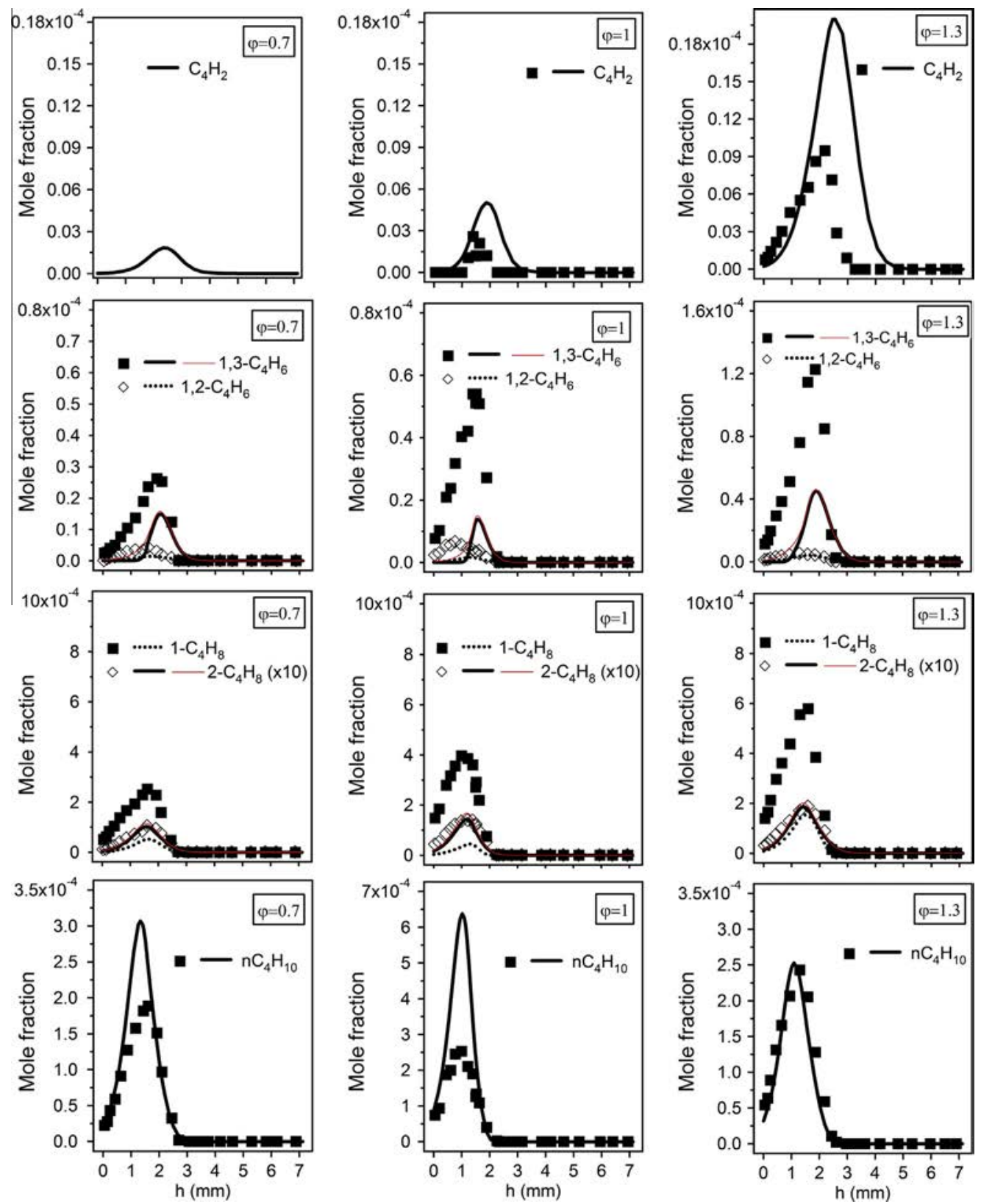

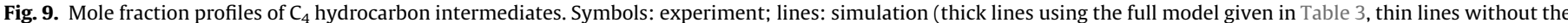
unimolecular initiations involving $\mathrm{C}-\mathrm{C}$ and $\mathrm{C}-\mathrm{O}$ breaking).

Figure 9 presents the $\mathrm{C}_{4}$ hydrocarbon intermediates, including 1,3-butadiyne $\left(\mathrm{C}_{4} \mathrm{H}_{2}\right)$, two isomers of $\mathrm{C}_{4} \mathrm{H}_{6}$ (1,3-butadiene $\left(1,3-\mathrm{C}_{4} \mathrm{H}_{6}\right)$ and 1,2-butadiene $\left.\left(1,2-\mathrm{C}_{4} \mathrm{H}_{6}\right)\right)$, two isomers of $\mathrm{C}_{4} \mathrm{H}_{8}$ (1-butene $\left(1-\mathrm{C}_{4} \mathrm{H}_{8}\right)$ and 2-butene $\left.\left(2-\mathrm{C}_{4} \mathrm{H}_{8}\right)\right)$, and $n$-butane $\left(n-\mathrm{C}_{4} \mathrm{H}_{10}\right)$. Among the $\mathrm{C}_{4} \mathrm{H}_{8}$ isomers and all $\mathrm{C}_{4}$ intermediates, $1-\mathrm{C}_{4} \mathrm{H}_{8}$ is present in largest amounts (contribution of $\sim 95 \%$ to the $\mathrm{C}_{4} \mathrm{H}_{8}$ isomers), with maximum mole fractions of $2.5 \times 10^{-4}$ $(\varphi=0.7), 4 \times 10^{-4}(\varphi=1.0)$ and $5.6 \times 10^{-4}(\varphi=1.3)$, respectively. It is followed by $n-\mathrm{C}_{4} \mathrm{H}_{10}$ and $1,3-\mathrm{C}_{4} \mathrm{H}_{6}$, in smaller amounts $\left(2.4 \times 10^{-4}\right.$ and $1.2 \times 10^{-4}$, respectively, at $\left.\varphi=1.3\right)$. Among $\mathrm{C}_{4} \mathrm{H}_{6}$ isomers, the mole fraction of $1,3-\mathrm{C}_{4} \mathrm{H}_{6}$ is the largest one. $\mathrm{C}_{4} \mathrm{H}_{2}$ is a minor species, formed for less than $10 \mathrm{ppm}$, even less than the GC detection limit in the fuel-lean flame. Other $C_{4}$ species (not shown in Fig. 9), e.g. 2-butyne $\left(2-\mathrm{C}_{4} \mathrm{H}_{6}\right)$, iso-butene $\left(\mathrm{iC}_{4} \mathrm{H}_{8}\right)$, and iso-butane $\left(\mathrm{iC}_{4} \mathrm{H}_{10}\right)$, were also detected, but with mole fractions lower than $7 \mathrm{ppm}$. The isomer identification is an interesting feature to compare between the present work and those of the literature. Kasper et al. [20] have also detected $n$-butane, 1-butene, 2-butene, and 1,3-butadiene in their THF flames using PI-MBMS. 1-Butene, 1,3-butadiene and diacetylene were also identified in the THF thermal decomposition studied by Lifshitz et al. [15]. Only 1-butene has been identified and quantified in the THF oxidation study by Dagaut et al. [19], using GC for analyzing the products formed in a JSR. The mole fraction profiles of $\mathrm{C}_{4} \mathrm{H}_{2}, 2-\mathrm{C}_{4} \mathrm{H}_{8}, 1$, $2-\mathrm{C}_{4} \mathrm{H}_{6}$ and $n-\mathrm{C}_{4} \mathrm{H}_{10}$ are well predicted, while those of the most abundant species, $1-\mathrm{C}_{4} \mathrm{H}_{8}$ and $1,3-\mathrm{C}_{4} \mathrm{H}_{6}$, are significantly underpredicted by the model, probably showing that an unknown pathway leading to 1-butene is missing in the mechanism.

Figure 10 presents the $C_{5}-C_{6}$ hydrocarbon intermediates, including two isomers of $\mathrm{C}_{5} \mathrm{H}_{10}$ (1-pentene $\left(1-\mathrm{C}_{5} \mathrm{H}_{10}\right)$ and 2-pentene $\left.\left(2-\mathrm{C}_{5} \mathrm{H}_{10}\right)\right)$, and benzene $\left(\mathrm{C}_{6} \mathrm{H}_{6}\right)$. These species were formed in small amounts $(<15 \mathrm{ppm})$. Among the $\mathrm{C}_{5} \mathrm{H}_{10}$ isomers, $1-\mathrm{C}_{5} \mathrm{H}_{10}$ 

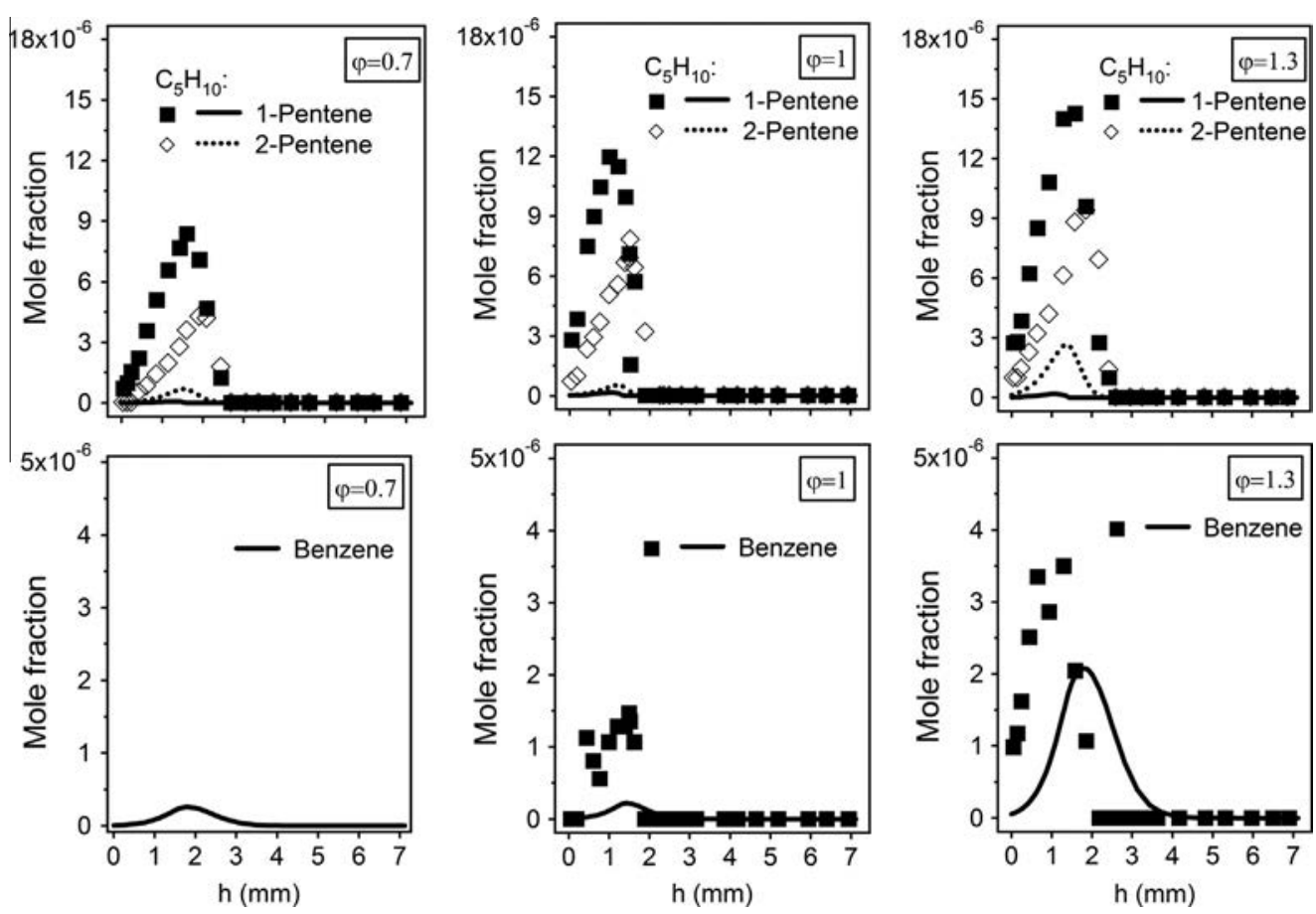

Fig. 10. Mole fraction profiles of $C_{5}-C_{6}$ hydrocarbon intermediates. Symbols: experiment; lines: simulation.

is more abundant than $2-\mathrm{C}_{5} \mathrm{H}_{10}$. In the THF flames of Kasper et al. [20], 1- $\mathrm{C}_{5} \mathrm{H}_{10}$ was also detected, but 2- $\mathrm{C}_{5} \mathrm{H}_{10}$ was not reported. Cyclic unsaturated hydrocarbons, important soot precursors, were detected with small mole fractions, less than $4 \mathrm{ppm}$ for benzene $\left(\mathrm{C}_{6} \mathrm{H}_{6}\right)$ and at trace level for 1,3-cyclopentadiene $\left(1,3-\mathrm{C}_{5} \mathrm{H}_{6}\right)$. This should make THF family potential "clean" bio-fuel considering soot formation. $C_{5}$ species products have not been presented in the THF oxidation study of Dagaut et al. [19]. Like for the major $\mathrm{C}_{4}$ species, the model also significantly underpredicts the formation of $\mathrm{C}_{5}$ species. Thanks to the addition of reactions related to fulvene, the prediction of benzene formation agrees within a factor 2 with the experimental measurements.

The mole fraction profiles of oxygenated intermediates are shown in Fig. 11 for the $C_{1}-C_{2}$ species and in Fig. 12 for $C_{3}-C_{5}$ species. Formaldehyde ( $\mathrm{HCHO}$, Fig. 11) is measured with the largest mole fractions. The mole fraction profiles of this compound reach their maxima of $2.7 \times 10^{-3}$ at $h \sim 1.6 \mathrm{~mm}(\varphi=0.7), 2.9 \times 10^{-3}$ at $h \sim 1.2 \mathrm{~mm}(\varphi=1.0)$, and $3.6 \times 10^{-3}$ at $h \sim 1.6 \mathrm{~mm}(\varphi=1.3)$. Other abundant oxygenated intermediates are acetaldehyde $\left(\mathrm{CH}_{3} \mathrm{CHO}\right.$; Fig. 11), propanal $\left(\mathrm{C}_{2} \mathrm{H}_{5} \mathrm{CHO}\right)$, acrolein $\left(\mathrm{C}_{2} \mathrm{H}_{3} \mathrm{CHO}\right)$ /furan $\left(\mathrm{C}_{4} \mathrm{H}_{4} \mathrm{O}\right)$ mixture (these two compounds were not separated experimentally by GC), and 2,3-dihydrofuran (2,3-DHF) (Fig. 12), with maximum mole fractions of $2.6 \times 10^{-3}, 2.0 \times 10^{-4}, 1.5 \times 10^{-4}$ and $1.2 \times 10^{-4}$, respectively, in the stoichiometric flame. Figure 12 shows that propanal is the most abundant species among $C_{3}-C_{5}$ oxygenated intermediates, and that 2,3-dihydrofuran is $\sim 4.4$ times more produced than 2,5-dihydrofuran. Under similar conditions, the ethanol flame previously reported by our group [24] led to about 20 times larger acetaldehyde mole fractions $\left(5.5 \times 10^{-3}\right.$ at $\varphi=1.0)$, with, however, formaldehyde mole fractions $\left(2.8 \times 10^{-3}\right.$ at $\varphi=1.0$ ) quite similar to those in the present THF flame. Acetaldehyde and formaldehyde mole fractions reach higher levels in the less diluted stoichiometric THF flame reported by Kasper et al. [20] than in the present work (by a factor of $\sim 3$ ). Nevertheless, the ratio of formaldehyde to acetaldehyde $(\sim 10)$ is similar in both studies at $\varphi=1$. In comparison with the studies of Dagaut et al. [19] and
Kasper et al. [20], the acetaldehyde/propanal ratio (approximately $\sim 1-1.5$ ) is quite similar at $\varphi=1$, despite the individual propanal and acetaldehyde mole fractions being different between the three studies.

In the present measurements, the quantification of ketene (Fig. 11) certainly has a large uncertainty, because this compound is very reactive and can significantly be lost in the sampling line before GC. In the recent work of Kasper et al. [20] using the EI-and PI-MBMS, ketene is measured in large amounts, similar to acetaldehyde. The flow-rate analysis for the consumption of THF (presented in the next section) shows that ketene is an important primary product. In the present work, acrolein and furan (Fig. 12) were not separated by GC. However, the signals from the GC-MS show that acrolein is more abundant than furan (by a factor of about 2-3). The same trend was observed in the measurements of Kasper et al. [20].

Other oxygenated intermediates, such as dimethylether (DME, $\mathrm{CH}_{3} \mathrm{OCH}_{3}$, Fig. 11), acetone $\left(\mathrm{CH}_{3} \mathrm{COCH}_{3}\right)$, 2,5-dihydrofuran (2,5DHF), 2-methyltetrahydrofuran (MTHF), cyclopropanecarboxaldehyde, 2-butenal (Fig. 12), and ethylene oxide (not shown here) are present in smaller concentrations. 2-Butenal was also identified in the THF flame reported by Kasper et al. [20]. However the presence of cyclopropanecarboxaldehyde in this last work could neither be confirmed nor excluded based on the flame-sampled PIE curves because of a complicated convolution of signals at $\mathrm{m} /$ $z=70$ [20]. However, this species was well identified and quantified in the present THF flames (Fig. 12). Kasper et al. [20] have also detected 2,3-dihydrofuran, 2,5-dihydrofuran, and MTHF in their THF flames. In the study of Dagaut et al. [19], only three oxygenated intermediates (formaldehyde, acetaldehyde, and propanal) were measured; others such as dihydrofurans were detected at trace levels.

The model very reasonably predicts the formation of oxygenated intermediates, especially for the two cancerogenic species (formaldehyde and acetaldehyde) (Fig. 11) that are present in very large concentrations. The overprediction of ketene mole 

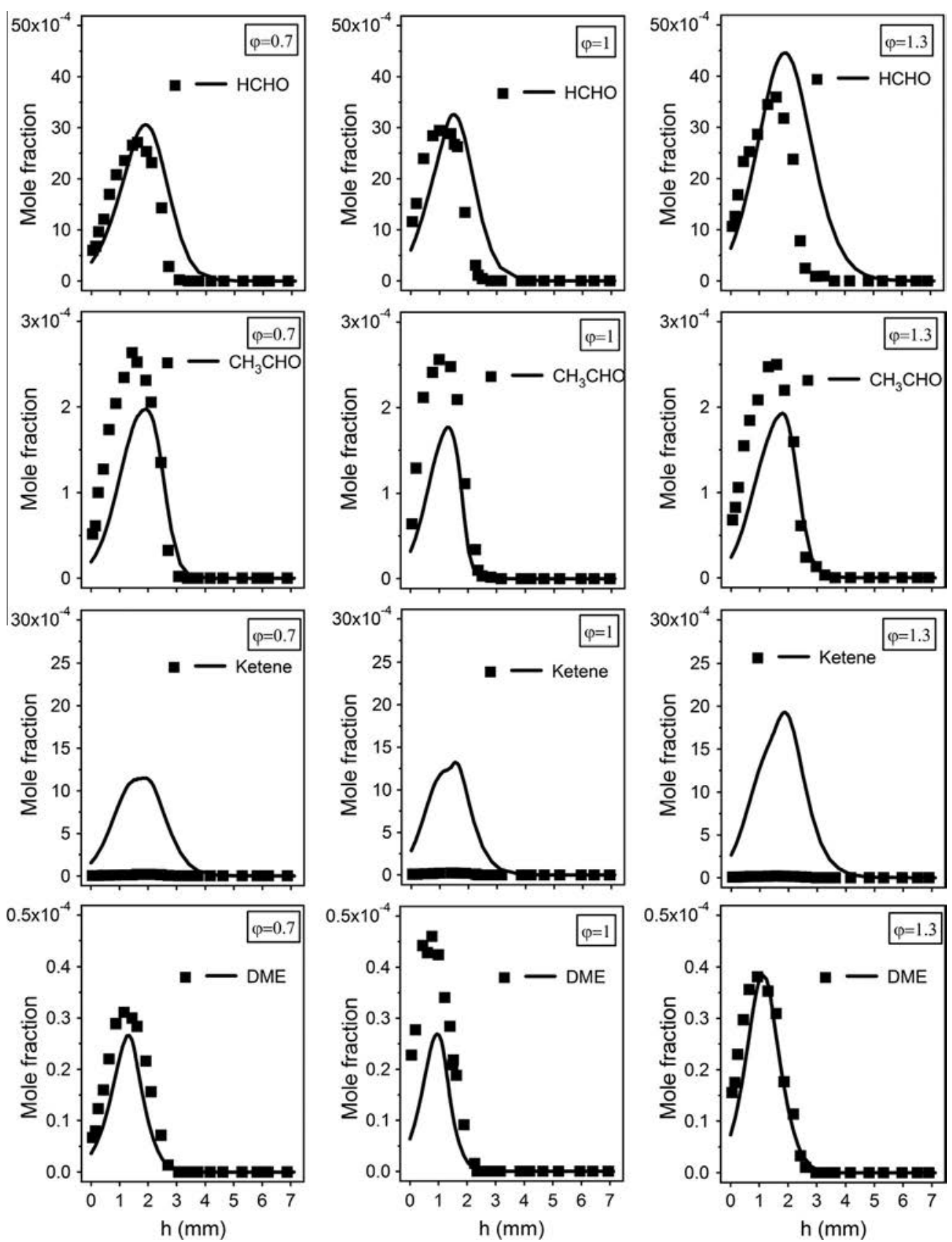

Fig. 11. Mole fraction profiles of $C_{1}-C_{2}$ oxygenated intermediates. Symbols: experiment; lines: simulation.

fraction profiles, is probably due to losses in the sampling line as discussed previously. Note that the present model predicts quite well the formation of this species under flame conditions of Kasper et al. [20] as presented in the next paragraph. It is interesting to note that the experimental formation of acrolein-furan mixture is lower at $\varphi=1.0$ than at $\varphi=0.7$ and 1.3 . The model well reflects this effect of equivalence ratio. The profile of mole fraction of both 2,5-DHF and 2,3-DHF is satisfactorily reproduced by the model. The modeling of the profiles of 2,3-DHF is significantly improved when the isomerizations of Fig. 4 are added. Concerning the formation of other obtained products, the profiles of cyclopropanecarboxaldehyde and MTHF are well reproduced by the model, whereas those of 2-butenal which is formed in low amounts (less than $10 \mathrm{ppm}$ ) are significantly overpredicted. However, under the flame conditions of Kasper et al. [20], the mole fraction of this species is satisfactorily reproduced by the model as presented hereafter.

In order to test the ability of the present kinetic model to reproduce data obtained in another independent experimental setup, we have simulated the structure of the fuel-rich THF flame $(\varphi=1.75$, Ar dilution $=30 \%, P=3.33 \mathrm{kPa}$ ) of Kasper et al. [20]. These data were measured by PI-MBMS, an analytical technique able to detect and quantify reactive species, such as radicals, ethenol, and ketene. Figures S3-S5 in the Supplemental Material show a comparison of these simulations with the experimental flame data at an equivalence ratio of 1.75. A globally correct agreement can be observed, for the mole fraction profiles of reactants and main products (Fig. S3) especially. The present model is able to capture, within the error range of MBMS measurements, the formation of primary species, including methyl radical, ketene, and 2-butenal (Fig. S4). 

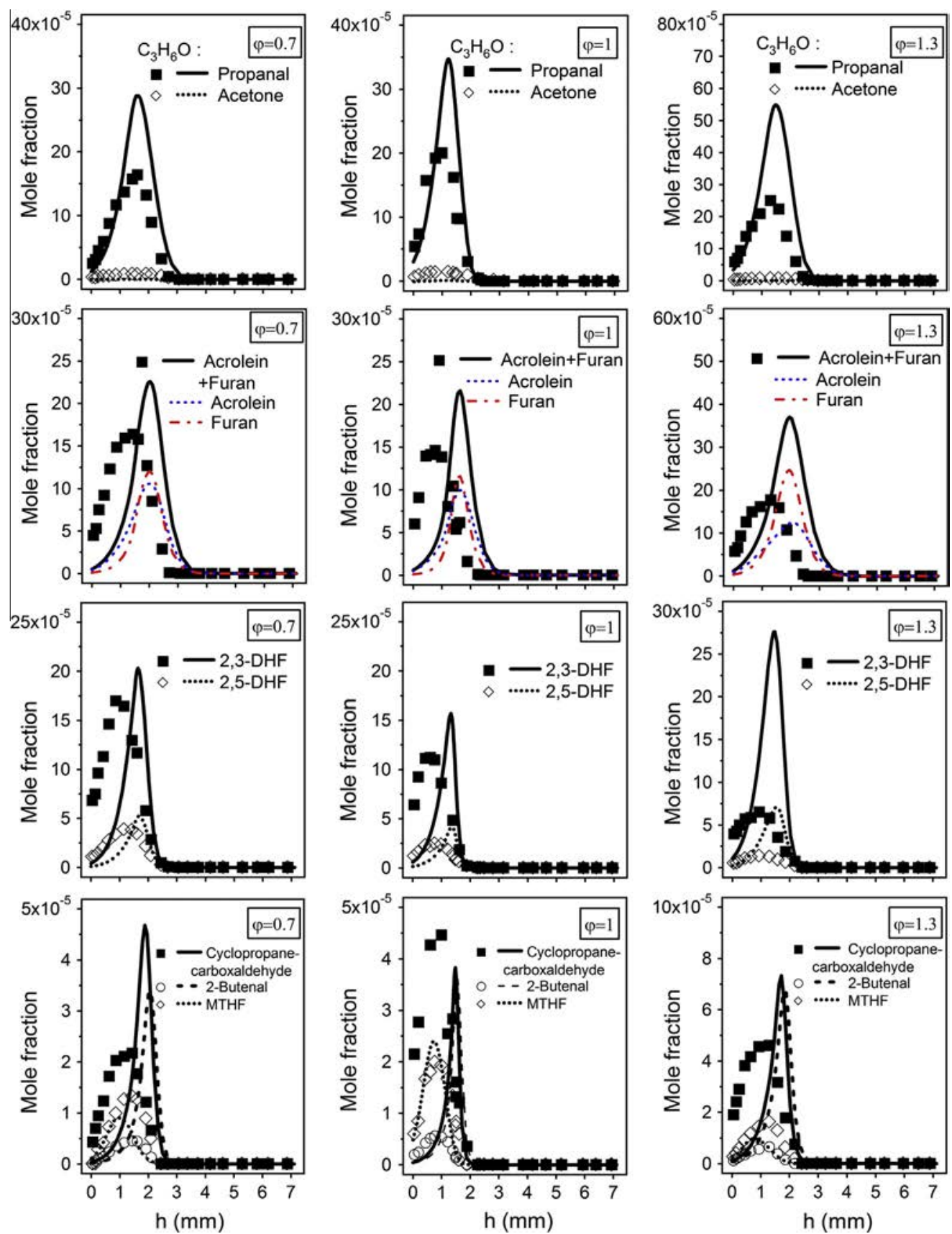

Fig. 12. Mole fraction profiles of $C_{3}-C_{5}$ oxygenated intermediates. Symbols: experiment; lines: simulation.

\subsection{Laminar burning velocities of THF at atmospheric pressure}

To our knowledge, no investigation on the adiabatic laminar burning velocities of THF in air has been published prior to the present study. Figure 13 reports the data (with error bars) for the adiabatic laminar burning velocities of THF-air mixtures measured on a flat flame burner at atmospheric pressure with temperatures of unburned gases ranging from 298 to $398 \mathrm{~K}$ and equivalence ratios ranging from 0.55 to 1.60 . As expected, these data show that the laminar burning velocity increases when increasing the initial temperature. For each temperature of the fresh gases, the maximum flame burning velocity occurs under conditions slightly richer $(\varphi=1.10)$ than stoichiometry.

Figure 13 shows that the model satisfactorily captures the effect of equivalence ratios and initial temperatures which considerably influence laminar burning velocities, although current simulations overpredict significantly the measured laminar burning velocities especially for elevated temperatures in lean and stoichiometric mixtures.

\subsection{Auto-ignition of THF in shock tube}

Figure 14 presents the experimental and modeling results obtained for the ignition delay times $\left(t_{\text {igni }}\right)$ of THF-oxygen-argon mixtures containing $0.25 \%, 0.5 \%$, and $1 \%$ of fuel for equivalence ratios of $0.5,1.0$ and 2.0. This figure shows that for all studied mixtures, ignition delay times decrease when temperature rises and varies exponentially vs. $1000 / T$. It can be noted that for a given temperature and equivalence ratio, ignition delay times increase with the decrease of fuel concentration from $1 \%$ to $0.25 \%$. For a given temperature and fuel concentration, ignition delay times increase when going from fuel-lean to fuel-rich conditions. 


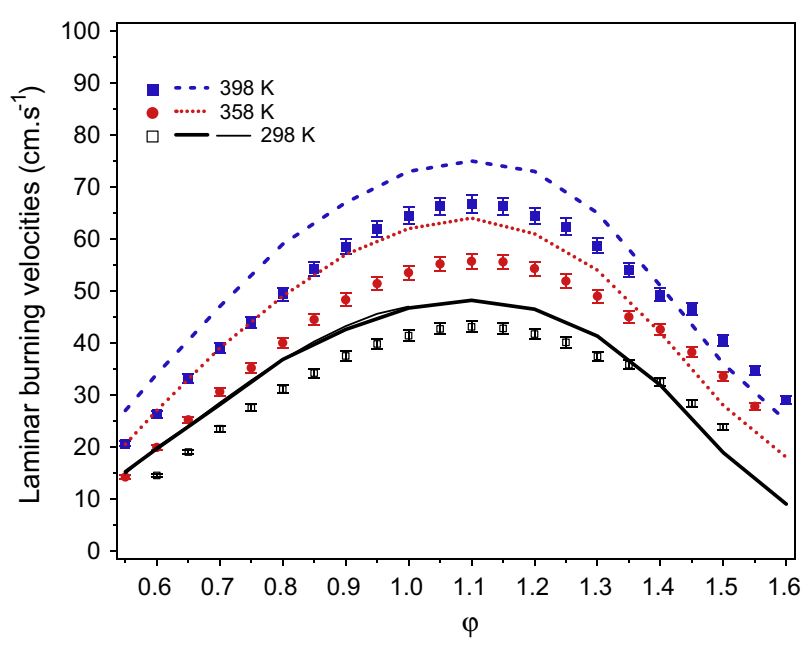

Fig. 13. Laminar burning velocities for THF-air flames at $101 \mathrm{kPa}$ ( $1 \mathrm{~atm})$. Symbols correspond to experiments; lines to simulations, thick lines using the full model given in Table 3, thin line without the unimolecular initiations involving $\mathrm{C}-\mathrm{C}$ and $\mathrm{C}-$ $\mathrm{O}$ breaking.

For fuel/oxygen mixtures, the determination of power dependences is often proposed from the overall statistical correlation between $t_{\text {igni }}$ and the gas concentrations shown in Eq. (4):

$t_{\text {igni }}=A \exp (\mathrm{E} / \mathrm{RT})[\mathrm{HC}]^{\mathrm{a}}\left[\mathrm{O}_{2}\right]^{\mathrm{b}}[\mathrm{Ar}]^{\mathrm{c}}$

where $A$ is the pre-exponential factor, $R$ the gas constant (in this work, in cal $\mathrm{K}^{-1} \mathrm{~mol}^{-1}$ ) and $\mathrm{E}$ the apparent "activation energy". For a restricted range of pressure and temperature, a, b and c are usually constant. Such a statistical correlation has been derived from the present experiments, but since the mole fraction of argon had small variations under the different initial conditions (from 93.5\% to $98.125 \%$ ) and seems not to affect the delay times, it was chosen to keep $c=0$. A multi-linear regression leads to the following relationship, with the concentrations behind the reflected shock wave in $\mathrm{mol} \mathrm{cm}{ }^{-3}$ :

$t_{\text {igni }}(\mathrm{s})=9.32 \times 10^{-24} \exp (80082 / \mathrm{RT})[\mathrm{THF}]^{0.22}\left[\mathrm{O}_{2}\right]^{-1.474}$

The statistical correlation of Eq. (5) shows a strong negative $\mathrm{O}_{2}$ power dependence, while the fuel power dependence is small, near 0.2 .

The current model reflects well the systematic trends observed and also satisfactorily reproduces the ignition delay time values, even if the predicted slope tends to be lower than the experimental ones for initial mole fractions smaller than $1 \%$.

\section{Discussion}

Below are discussed the main flows of consumption of THF and the formation pathways of the important products measured under low-pressure stoichiometric flame conditions. Figure 15 presents the simulated main fuel consumption pathways at a distance of $1.41 \mathrm{~mm}$ above the burner under the conditions of Figs. 5-12. These correspond to a simulated temperature of $1120 \mathrm{~K}$ and a 90\% THF conversion.

Under these conditions, THF is mainly (76\%) consumed by $\mathrm{H}-$ abstractions from the $\mathrm{C} 2$ or $\mathrm{C} 5$ positions of THF (the carbon atoms bound to the oxygen atom, see Fig. 1) by flame propagating radicals, such as $\mathrm{H}, \mathrm{OH}$, and $\mathrm{O}$, to produce tetrahydrofur-2-yl radical (THF-yl-2). H-abstractions from the $\mathrm{C} 3$ or $\mathrm{C} 4$ positions of THF that yield tetrahydrofur-3-yl radical (THF-yl-3) have a lower contribution to the fuel consumption ( $24 \%$ ) than the first mentioned pathway. The difference in the contribution to fuel consumption between these two main channels is consistent with the difference in energy of $\mathrm{C}-\mathrm{H}$ bonds between the different positions in the THF molecule. As is shown in Fig. 1, the energy of $\mathrm{C}-\mathrm{H}$ bonds in the positions $\mathrm{C} 2$ and $\mathrm{C} 5$ is lower $\left(93.9 \mathrm{kcal} \mathrm{mol}^{-1}\right)$ than that of $\mathrm{C}-\mathrm{H}$ bonds in the positions C3 and C4 (98.3 kcal mol $\left.{ }^{-1}\right)$. The contribution of other channels to the THF consumption under these conditions is very small $(<1 \%)$.

Under the conditions of the flow-rate analysis, the THF-yl-2 radical reacts mainly by $\mathrm{C}-\mathrm{O}$ bond $\beta$-scission leading to the formation of $\mathrm{R} \mathrm{CC}_{4} \mathrm{H}_{7} \mathrm{OA}$ radical. Minor consumption channels of THF-yl2 radical are decompositions by $\mathrm{C}-\mathrm{C}$ bond $\beta$-scission yielding

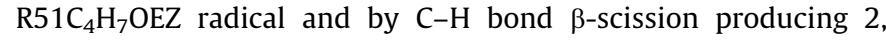
3-DHF, as well as combination with methyl radicals to give

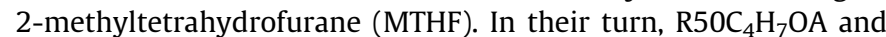

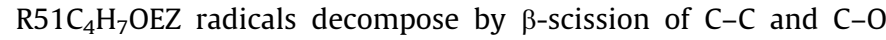
bonds, respectively, to produce ethylene and the resonance-stabilized $\mathrm{CH}_{2} \mathrm{CHO}$ radical. This is the major source $(\sim 80 \%)$ of ethylene which was spotted as the most abundant intermediate species. The ethylene mole fraction profiles are well reproduced by the model. Several compounds, such as ketene, carbon monoxide, acetaldehyde, formaldehyde, and propanal, are produced from the $\mathrm{CH}_{2} \mathrm{CHO}$ radical. About $85 \%, 60 \%$, and $20 \%$ of the formation of ketene, acetaldehyde and formaldehyde, respectively, come from these reaction channels. Formaldehyde can also be formed directly from the decomposition of THF by another route $(\sim 30 \%$ of its relative

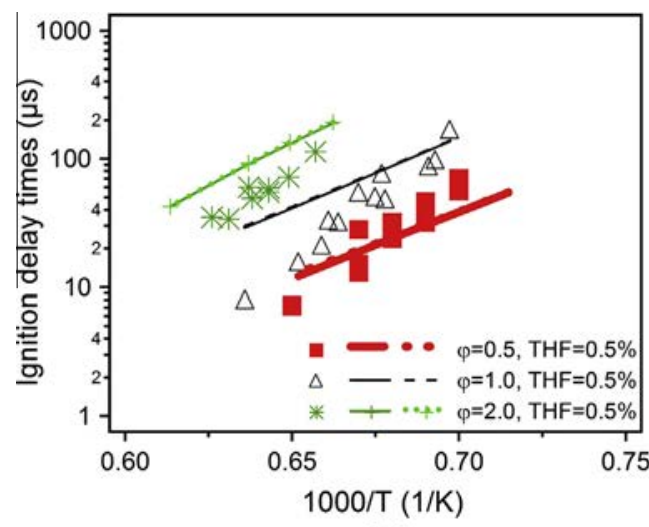

(a)

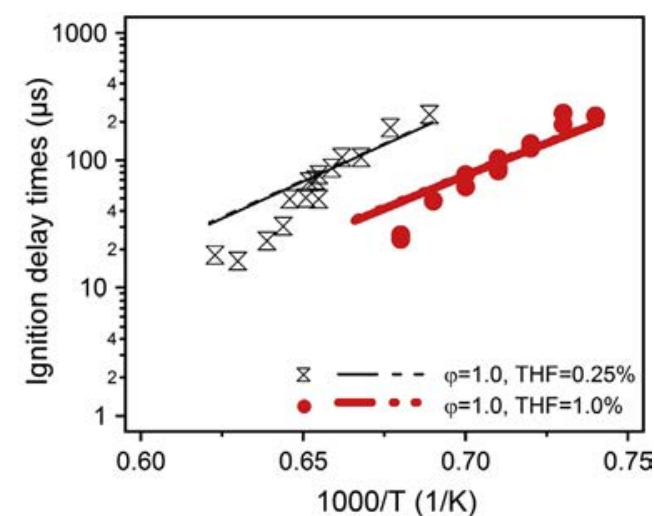

(b)

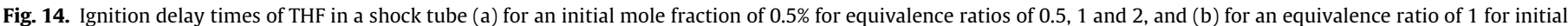

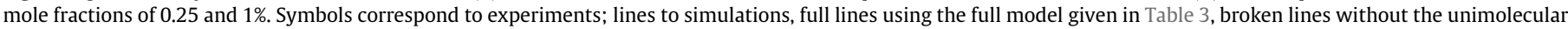
initiations involving $\mathrm{C}-\mathrm{C}$ and $\mathrm{C}-\mathrm{O}$ breaking. 


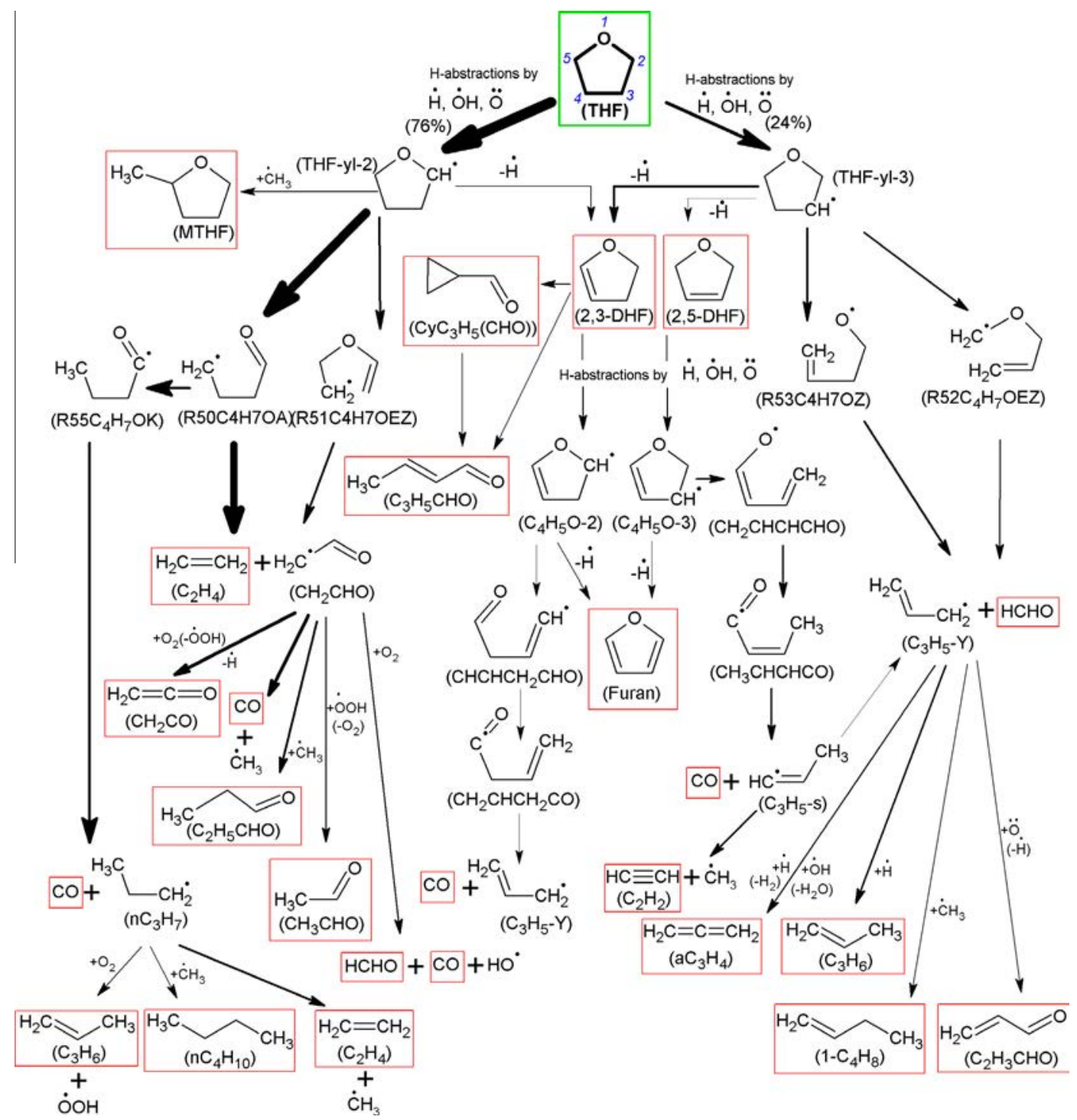

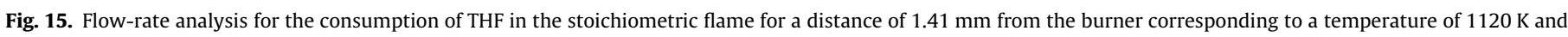

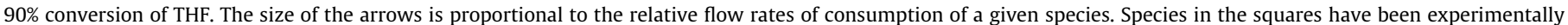
detected.

formation flow) that will be presented in the analysis below. The formaldehyde formation is also due in a large extent to several other reactions. As is shown in Figs. 11 and 12, the mole fraction profiles of acetaldehyde, formaldehyde, and propanal are very well reproduced by the model, demonstrating that their main formation channels are well taken into account.

A second channel of $\mathrm{R}^{2} 0 \mathrm{C}_{4} \mathrm{H}_{7} \mathrm{OA}$ radical consumption is an isomerization by internal transfer of hydrogen atom between the $\mathrm{C} 1$ and $\mathrm{C} 4$ positions. This reaction leads to the formation of $\mathrm{R}_{55 \mathrm{C}_{4}}$ $\mathrm{H}_{7} \mathrm{OK}$ radical which then decomposes into $\mathrm{CO}$ and $n$-propyl radical $\left(n \mathrm{C}_{3} \mathrm{H}_{7}\right) . n \mathrm{C}_{3} \mathrm{H}_{7}$ radical is consumed to yield $\mathrm{C}_{2} \mathrm{H}_{4}$ and $\mathrm{CH}_{3}$ radicals by $\beta$-scission, or propene and $\mathrm{HO}_{2}$ radical by reaction with $\mathrm{O}_{2}$, or $n$-butane by combination with $\mathrm{CH}_{3}$ radicals. More than $90 \%$ of the formation of $\mathrm{C}_{3} \mathrm{H}_{6}$ and $n$-butane comes from these reaction pathways. Note that these two intermediates were measured in large amounts and their mole fraction profiles are reproduced satisfactorily by the model.

Under the conditions of the flow-rate analysis, the THF-yl-3 radical reacts by $\beta$-scission of $\mathrm{C}-\mathrm{O}, \mathrm{C}-\mathrm{C}$, or $\mathrm{C}-\mathrm{H}$ bonds to produce $\mathrm{R}_{53} \mathrm{C}_{4} \mathrm{H}_{7} \mathrm{OZ}, \mathrm{R}_{52} \mathrm{C}_{4} \mathrm{H}_{7} \mathrm{OEZ}$ radicals, or dihydrofurans (2,3-DHF and 2,5-DHF), respectively. Both $\mathrm{R}_{5} 3 \mathrm{C}_{4} \mathrm{H}_{7} \mathrm{OZ}$ and $\mathrm{R} 52 \mathrm{C}_{4} \mathrm{H}_{7} \mathrm{OEZ}$ radicals then completely decompose into formaldehyde and the resonance stabilized allyl radical $\left(\mathrm{C}_{3} \mathrm{H}_{5}-\mathrm{Y}\right)$. As it is mentioned in the previous paragraph, about $30 \%$ of the formation of $\mathrm{HCHO}$ comes from this reaction pathway. Several products can be produced from $\mathrm{C}_{3} \mathrm{H}_{5}-\mathrm{Y}$ radical, such as allene, propene, 1-butene, acrolein, and benzene. For unknown reasons, while the formation of allene, propene and acrolein/furan mixture is well-reproduced, 1-butene is the only product deriving from $\mathrm{C}_{3} \mathrm{H}_{5}-\mathrm{Y}$ radical to be incorrectly predicted. Benzene is formed for about $60 \%$ from fulvene which is produced mainly from the recombination of $\mathrm{C}_{3} \mathrm{H}_{5}-\mathrm{Y}$ with propargyl $\left(\mathrm{C}_{3} \mathrm{H}_{3}\right)$ radicals.

Dihydrofurans (2,3-DHF and 2,5-DHF) are mainly consumed by $\mathrm{H}$-abstractions with $\mathrm{H}, \mathrm{OH}$, and $\mathrm{O}$ to give the dihydrofuryl radicals $\left(\mathrm{C}_{4} \mathrm{H}_{5} \mathrm{O}-2\right.$ and $\left.\mathrm{C}_{4} \mathrm{H}_{5} \mathrm{O}-3\right)$. The reactions of both $\mathrm{C}_{4} \mathrm{H}_{5} \mathrm{O}-2$ and $\mathrm{C}_{4} \mathrm{H}_{5} \mathrm{O}-$ 3 radicals are an important part of the mechanism of furan oxidation that we have previously reported [25]. These radicals are mainly consumed by $\beta$-scissions of $\mathrm{C}-\mathrm{H}$ or $\mathrm{C}-\mathrm{O}$ bonds to give respectively furan or the $\mathrm{CH}_{2} \mathrm{CHCHCHO}$ and $\mathrm{CHCHCH}_{2} \mathrm{CHO}$ radicals, which are then consumed by isomerization followed by $\mathrm{CO}$ elimination. $\mathrm{CO}$ is an important primary product of the combustion of cyclic ethers. It is formed from several decomposition pathways 


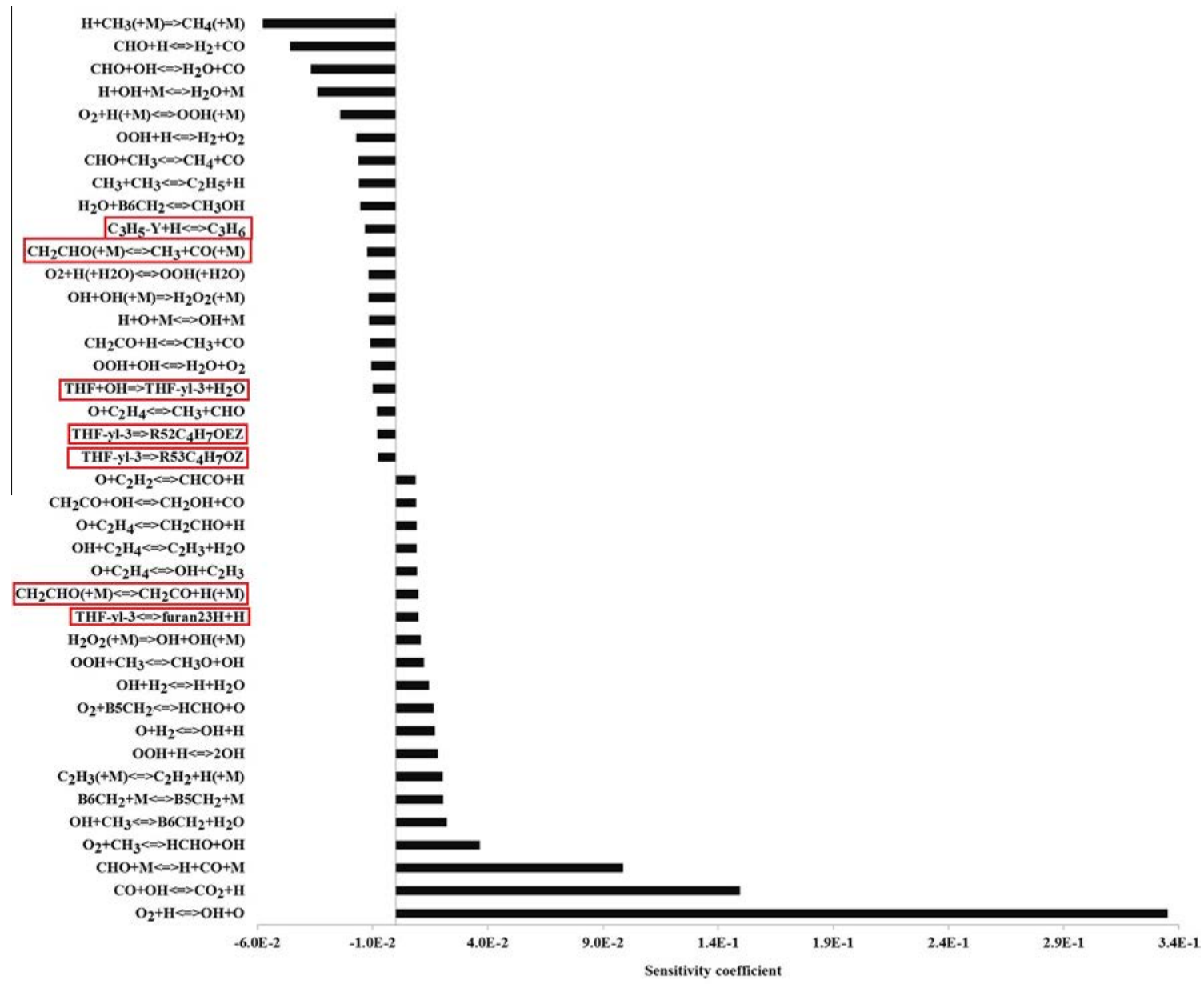

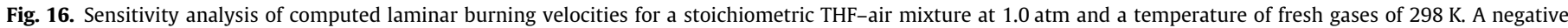
coefficient corresponds to a reaction which reduces the computed laminar burning velocity and vice versa. Reactions in the squares are reactions present in Table 3.

in the flow-rate analysis (Fig. 15). The model reproduces very well the formation of this major product (Fig. 6).

The reaction pathways consuming THF were also analyzed in the fuel-lean and fuel-rich THF flames, and similar reactions are involved with small differences in their respective importance. For instance, when the equivalence ratio decreases, the importance of reactions involving oxygenated radicals such as $\mathrm{OH}$ radicals or O-atoms is enhanced, while that of reactions involving non-oxygenated radicals such as $\mathrm{H}$-atoms or $\mathrm{CH}_{3}$ radicals is lowered.

To identify the reactions of importance for modeling adiabatic laminar burning velocities of THF in air, a sensitivity analysis is plotted in Fig. 16 for a temperature of fresh gases of $298 \mathrm{~K}$. This

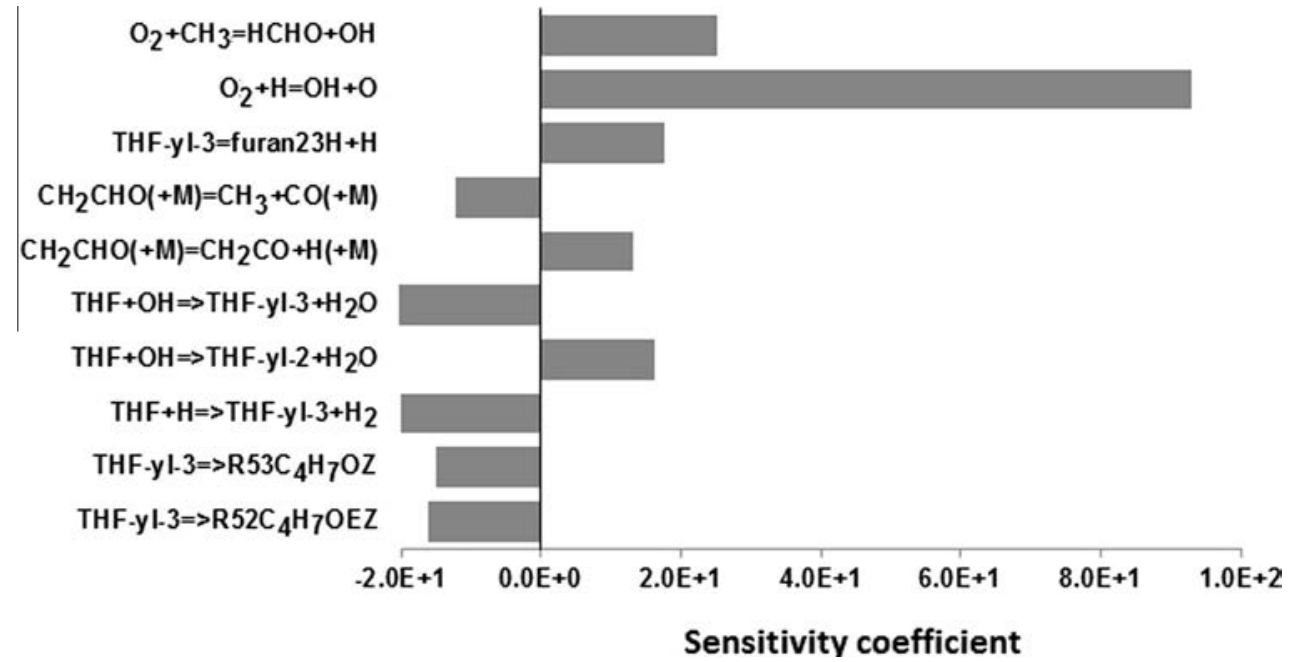

Fig. 17. Sensitivity analysis on $\mathrm{OH}^{*}$ radicals for ignition delay time at $\varphi=1.0\left(1 \% \mathrm{THF} / 5.5 \% \mathrm{O}_{2} / 93.5 \% \mathrm{Ar}\right), 1423 \mathrm{~K}$, and 8.58 atm. 
figure shows that only a few reactions of Table 3, those of THF-yl-3 radicals, are listed among sensitive ones. Therefore the cause of the disagreement between experiments and simulations for laminar burning velocities has certainly to be found in the reaction base, probably reactions of small oxygenated species.

To better know the reactions of influence to model auto-ignition delay times, Fig. 17 presents a sensitivity analysis concerning $\mathrm{OH}^{*}$ radicals. Among the reactions of Table 3, the most sensitive ones are those involving THF-yl-3 radicals, the same reactions which are of influence for simulating laminar burning velocities as is shown in Fig. 16. THF-yl-3 radicals can notably decompose to give 2,3-DHF and $\mathrm{H}$-atoms, which are involved in $\mathrm{H}+\mathrm{O}_{2}=\mathrm{O}+\mathrm{OH}$, the very promoting branching step. This $\mathrm{C}-\mathrm{H}$ bond $\beta$-scission has then a promoting effect. On the other side, the competing $\mathrm{C}-\mathrm{O}$ and $\mathrm{C}-\mathrm{C}$ bond $\beta$-scissions leading to $\mathrm{R}_{53} \mathrm{C}_{4} \mathrm{H}_{7} \mathrm{OZ}$ and $\mathrm{R} 52 \mathrm{C}_{4} \mathrm{H}_{7} \mathrm{OEZ}$ radicals have an inhibiting effect, which is also due to the fact that these radicals are a source of the resonance stabilized allyl radical $\left(\mathrm{C}_{3} \mathrm{H}_{5}-\mathrm{Y}\right)$. This last reason finally explains which the formations of THF-yl-3 radicals by $\mathrm{H}$-abstractions have an inhibiting effect.

As is shown in Fig. 15, whereas the reactions going via unimolecular initiation involving $\mathrm{C}-\mathrm{C}$ and $\mathrm{C}-\mathrm{O}$ bond breaking had a significant influence under pyrolysis conditions [16], the influence on ignition delays times of this part of the mechanism (reactions $3-9$ and $80-127$ in Table 3 ) is very limited: a very slight inhibiting effect at the highest temperatures at an equivalence ratio of 1 and $1 \%$ initial mole fraction. Concerning flame structures, the only effect of these reactions is a very slight increase of the profiles of 2-butene and of those of butadiene in the early zone of the flames (see Fig. 9). As shown in Fig. 13, the influence of these reactions on modeling laminar flame velocities is also negligible.

\section{Summary and conclusion}

This paper presents new experimental results for the combustion of tetrahydrofuran (THF): chemical structures of low-pressure laminar premixed flame, laminar burning velocities obtained under atmospheric pressure, and ignition delay times measured in a shock tube under high temperatures. During the low-pressure flame experiments, about 40 stable species were identified and quantified as a function of distance to the burner. A large number of selected important species have been presented, discussed and compared to those in previous studies reported in the literature. Among all intermediate species measured in the flames of this saturated cyclic ether, the production of ethylene is the largest.

THF combustion produces a significantly lower maximum mole fraction of acetaldehyde than those obtained during the combustion of ethanol under similar conditions, while formaldehyde formation is quite comparable for both fuels. Despite that the structure of laminar premixed low-pressure THF flames investigated using MBMS was already reported in the literature, the information from another independent quantitative measurement such as GC was very valuable to confirm species identification and to unambiguously identify the respective isomers.

The obtained experimental results have been used to develop a new mechanism for THF combustion. This new model can satisfactorily reproduce most of the products measured when probing the chemical structure of the THF flame, as well as ignition delay time and laminar burning velocity of this fuel. While the channels leading to $C_{4}$ or $C_{5}$ hydrocarbons certainly require to be more thoroughly investigated, the present model can be used as a basis to study the combustion of potential new bio-fuels, such as substituted tetrahydrofurans.

The model predicts correctly ignition delay times obtained in a shock tube, but it overpredicts significantly the measured laminar burning velocities, for elevated temperatures especially. The influence of the initiation reactions by breaking a $\mathrm{C}-\mathrm{C}$ or a $\mathrm{C}-\mathrm{O}$ bond, which was proposed in literature and had a significant impact under pyrolysis conditions, has been found negligible here under all studied conditions.

\section{Acknowledgments}

This work was funded by the European Commission through the "Clean ICE" Advanced Research Grant of the European Research Council and supported by the COST Action CM0901. This work was Granted access to the HPC resources of CINES under the allocation c2014087249 made by GENCI.

\section{References}

[1] M. Thewes, M. Muether, S. Pischinger, M. Budde, A. Brunn, A. Sehr, P. Adomeit, Jlankermayer, Energy Fuels 25 (2011) 5549-5561.

[2] J.M. Simmie, J. Phys. Chem. A 116 (2012) 4528-4538.

[3] L.S. Tran, B. Sirjean, P.-A. Glaude, R. Fournet, F. Battin-Leclerc, Energy 43 (2012) 4-18.

[4] G.W. Huber, S. Iborra, A. Corma, Chem. Rev. 106 (2006) 4044-4098.

[5] W. Yang, A. Sen, ChemSusChem 3 (2010) 597-603.

[6] F.M.A. Geilen, B. Engendahl, A. Harwardt, W. Marquardt, J. Klankermayer, W. Leitner, Angew. Chem. - Int. Edit. 49 (2010) 5510-5514.

[7] J.-P. Lange, E. van der Heide, J. van Buijtenen, R. Price, ChemSusChem 5 (2012) $150-166$

[8] T.W. Rudolph, J.J. Thomas, Biomass 16 (1988) 33-49.

[9] S.F. Paul, Prepr. Symp. Am. Chem. Soc. Div. Fuel Chem. 43 (1998) 373-377.

[10] Department of Energy, Federal Register, 64, 94 (1999) 26822-26829.

[11] W.R. Leppard, SAE Trans. 98 (1989) 879-904.

[12] O. Herbinet, F. Battin-Leclerc, S. Bax, H.L. Gall, P.-A. Glaude, R. Fournet, Z. Zhou, L. Deng, H. Guo, M. Xie, F. Qi, Phys. Chem. Chem. Phys. 13 (2011) 296-308.

[13] C.H. Klute, W.D. Walters, J. Am. Chem. Soc. 68 (1946) 506-511.

[14] G. Mcdonald, N.M. Lodge, W.D. Walters, J. Am. Chem. Soc. 73 (1951) $1757-$ 1760.

[15] A. Lifshitz, M. Bidani, S. Bidani, J. Phys. Chem. 90 (1986) 3422-3429.

[16] M. Verdicchio, B. Sirjean, L.S. Tran, P.-A. Glaude, F. Battin-Leclerc, Proc. Combust. Inst. 35 (2015) 533-541.

[17] M.J. Molera, A. Couto, J.A. García-Domínguez, Int. J. Chem. Kinet. 20 (1988) 673-685.

[18] W.R. Leppard, SAE Trans. 100 (1991) 589-604.

[19] P. Dagaut, M. McGuinness, J.M. Simmie, M. Cathonnet, Combust. Sci. Technol. 135 (1998) 3-29.

[20] T. Kasper, A. Lucassen, A.W. Jasper, W. Li, P.R. Westmoreland, K. KohseHöinghaus, B. Yang, J. Wang, T.A. Cool, N. Hansen, Z. Physik Chem. 225 (2011) $1237-1270$.

[21] Y. Uygun, S. Ishihara, H. Olivier, Combust. Flame 161 (2014) 2519-2530.

[22] J.M. Simmie, H.J. Curran, J. Phys. Chem. A 113 (2009) 5128-5137.

[23] E. Pousse, Z.Y. Tian, P.A. Glaude, R. Fournet, F. Battin-Leclerc, Combust. Flame 157 (2010) 1236

[24] L.-S. Tran, P.-A. Glaude, R. Fournet, et F. Battin-Leclerc, Energy Fuels 27 (2013) 2226-2245.

[25] D. Liu, C. Togbé, L.-S. Tran, D. Felsmann, P. Oßwald, P. Nau, J. Koppmann, A. Lackner, P.-A. Glaude, B. Sirjean, R. Fournet, F. Battin-Leclerc, K. KohseHöinghaus, Combust. Flame 161 (2014) 748-765.

[26] U. Bonne, T. Grewer, H.G. Wagner, Z. Physik. Chem. 26 (1960) 93-110.

[27] L.S. Tran, B. Sirjean, P.-A. Glaude, K. Kohse-Höinghaus, F. Battin-Leclerc, Proc. Combust. Inst. 35 (2015) 1735-1743.

[28] L.H.P. De Goey, A. Van Maaren, R.M. Quax, Combust. Sci. Technol. 92 (1993) 201-207.

[29] P. Dirrenberger, H. Le Gall, R. Bounaceur, O. Herbinet, P.-A. Glaude, A. Konnov, F. Battin-Leclerc, Energ. Fuels 25 (2011) 3875-3884

[30] P. Dirrenberger, P-A. Glaude, R. Bounaceur, H. Le Gall, A. Pires da Cruz, A. Konnov, F. Battin-Leclerc, Fuel 115 (2014) 162-169.

[31] B. Sirjean, F. Buda, H. Hakka, P.A. Glaude, R. Fournet, V. Warth, F. Battin-Leclerc, M. Ruiz-Lopez, Proc Combust. Inst. 31 (2007) 277-284.

[32] H. Bennadji, L. Coniglio, F. Billaud, R. Bounaceur, V. Warth, P.-A. Glaude, F. Battin-Leclerc, Int. J. Chem. Kin. 43 (2011) 204-218.

[33] R.J. Kee, F.M. Rupley, J.A. Miller, Sandia Laboratories Report. No. SAND-898009B, 1993.

[34] B. Sirjean, R. Fournet, P.-A. Glaude, F. Battin-Leclerc, W. Wang, M.A. Oehlschlaeger, J. Phys. Chem. A 117 (2013) 1371-1392. 
[35] V. Warth, N. Stef, P.A. Glaude, F. Battin-Leclerc, G. Scacchi, G.M. Côme, Combust. Flame 114 (1998) 81-102.

[36] F. Buda, R. Bounaceur, V. Warth, P.A. Glaude, R. Fournet, F. Battin-Leclerc, Combust. Flame 142 (2005) 170-186.

[37] B. Heyberger, N. Belmekki, V. Conraud, P.A. Glaude, R. Fournet, F. BattinLeclerc, Int. J. Chem. Kin. 36 (2002) 666-677.

[38] D.L. Allara, R.J. Shaw, J. Phys. Chem. Ref. Data 9 (1980) 523-559.

[39] A.M. Dean, J.W. Bozzelli, Combustion Chemistry of Nitrogen, in Gas-phase Combustion Chemistry. NewYork, W.C. Gardiner, Jr., 2000.

[40] F. Battin-Leclerc, V. Warth, R. Bounaceur, B. Husson, O. Herbinet, P.A. Glaude, Proc. Combust. Inst. 35 (2015) 349-356.

[41] H.A. Gueniche, J. Biet, P.A. Glaude, R. Fournet, F. Battin-Leclerc, Fuel 88 (2009) 1388-1393.

[42] B. Husson, M. Ferrari, O. Herbinet, S.S. Ahmed, P.A. Glaude, F. Battin-Leclerc, Proc. Combust. Inst. 34 (2013) 325-333.

[43] K. Moshammer, S. Vranckx, H.K. Chakravarty, P. Parab, R.X. Fernandes, K. Kohse-Höinghaus, Combust. Flame 160 (2013) 2729-2743.

[44] W. Li, M.E. Law, P.R. Westmoreland, T. Kasper, N. Hansen, K. Kohse-Höinghaus, Combust. Flame 158 (2011) 2077-2089.

[45] C. Muller, V. Michel, G. Scacchi, G.M. Come, J. Chem. Phys. 92 (1995) 11541177.

[46] S.W. Benson, Thermochemical Kinetics, second ed., Wiley, New York, 1976.

[47] M.J. Frisch, G.W. Trucks, H.B. Schlegel, G.E. Scuseria, M.A. Robb, J.R. Cheeseman, G. Scalmani, V. Barone, B. Mennucci, G.A. Petersson, H. Nakatsuji, M. Caricato, X. Li, H.P. Hratchian, A.F. Izmaylov, J. Bloino, G. Zheng, J.L. Sonnenberg, M.
Hada, M. Ehara, K. Toyota, R. Fukuda, J. Hasegawa, M. Ishida, T. Nakajima, Y. Honda, O. Kitao, H. Nakai, T. Vreven, J.A. Montgomery, J.E. Peralta, F. Ogliaro, M. Bearpark, J.J. Heyd, E. Brothers, K.N. Kudin, V.N. Staroverov, R. Kobayashi, J. Normand, K. Raghavachari, A. Rendell, J.C. Burant, S.S. Iyengar, J. Tomasi, M. Cossi, N. Rega, J.M. Millam, M. Klene, J.E. Knox, J.B. Cross, V. Bakken, C. Adamo, J. Jaramillo, R. Gomperts, R.E. Stratmann, O. Yazyev, A.J. Austin, R. Cammi, C. Pomelli, J.W. Ochterski, R.L. Martin, K. Morokuma, V.G. Zakrzewski, G.A. Voth, P. Salvador, J.J. Dannenberg, S. Dapprich, A.D. Daniels, Farkas, J.B. Foresman, J.V. Ortiz, J. Cioslowski, D.J. Fox, in, Wallingford CT, 2009.

[48] J.R. Barker, MultiWell-2013 Software, 2013, University of Michigan, Ann Arbor <http://aoss.engin.umich.edu/multiwell/>.

[49] J. Pfaendtner, X. Yu, L.J. Broadbelt, Theor. Chem. Acc. 118 (2007) 881-898.

[50] V. Mokrushin, W. Tsang, ChemRate, version 1.5.8, Gaithersburg, MD 20899, USA, 2009.

[51] C. Eckart, Phys. Rev. 35 (1930) 1303-1309.

[52] Z. Tian, T. Yuan, R. Fournet, P.-A. Glaude, B. Sirjean, F. Battin-Leclerc, K. Zhang, F. Qi, Combust. Flame 158 (2011) 756-773.

[53] A. Lifshitz, M. Bidani, S. Bidani, J. Phys. Chem. 93 (1989) 1139-1144.

[54] F. Dubnikova, A. Lifshitz, J. Phys. Chem. A 106 (2002) 1026-1034.

[55] L.-S. Tran, C. Togbé, D. Liu, D. Felsmann, P. Oßwald, P.-A. Glaude, R. Fournet, B. Sirjean, F. Battin-Leclerc, K. Kohse-Höinghaus, Combust. Flame 161 (2013) $766-779$.

[56] C. Togbé, L.-S. Tran, D. Liu, D. Felsmann, P. Oßwald, P.-A. Glaude, B. Sirjean, R. Fournet, F. Battin-Leclerc, K. Kohse-Höinghaus, Combust. Flame 161 (2013) 780-797. 